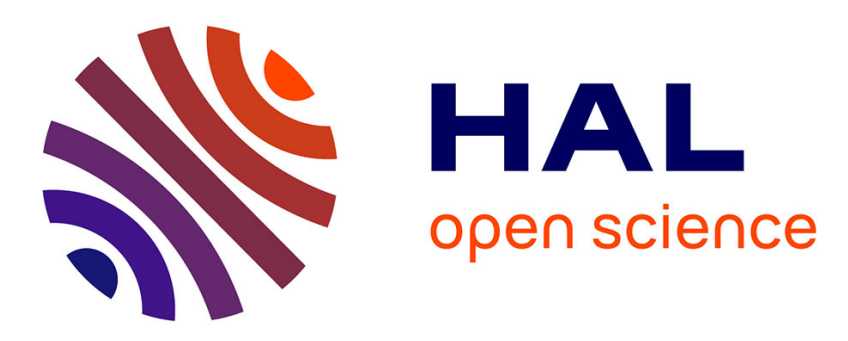

\title{
Multivalued robust tracking control of fully actuated Lagrange systems: Continuous and discrete-time algorithms
}

Felix Miranda-Villatoro, Bernard Brogliato, Fernando Castaños

\section{- To cite this version:}

Felix Miranda-Villatoro, Bernard Brogliato, Fernando Castaños. Multivalued robust tracking control of fully actuated Lagrange systems: Continuous and discrete-time algorithms. IEEE Transactions on Automatic Control, 2017, 62 (9), pp.4436-4450. 10.1109/TAC.2017.2662804 . hal-01254303

\section{HAL Id: hal-01254303 \\ https://hal.inria.fr/hal-01254303}

Submitted on 12 Jan 2016

HAL is a multi-disciplinary open access archive for the deposit and dissemination of scientific research documents, whether they are published or not. The documents may come from teaching and research institutions in France or abroad, or from public or private research centers.
L'archive ouverte pluridisciplinaire HAL, est destinée au dépôt et à la diffusion de documents scientifiques de niveau recherche, publiés ou non, émanant des établissements d'enseignement et de recherche français ou étrangers, des laboratoires publics ou privés. 


\title{
Multivalued robust tracking control of fully actuated Lagrange systems: Continuous and discrete-time algorithms.
}

\author{
Felix A. Miranda-Villatoro ${ }^{\dagger}$, Bernard Brogliato ${ }^{\ddagger}$, and Fernando Castaños ${ }^{\dagger}$ \\ ${ }^{\dagger}$ Automatic Control Department, CINVESTAV-IPN, 2508 av. Instituto \\ Politécnico Nacional, 07360, Mexico City, Mexico. \\ ${ }^{\ddagger}$ INRIA Grenoble Rhône-Alpes, University of Grenoble-Alpes, Inovalleé, 655 av. \\ de l'Europe, 38334, Saint-Ismier, France.
}

January 11, 2016

\begin{abstract}
In this paper the robust trajectory tracking problem of a class of nonlinear systems described by the Euler-Lagrange equations of motion is studied. We start considering a plant under the effects of an unknown external perturbation and also with uncertainties on its parameters. After that a class of passivity-based multivalued control laws is proposed and the well-posedness together with the stability of the closed-loop are established in the continuous-time setting. The discrete-time version of the plant and the controller are studied and well-posedness together with stability results are obtained, using the so-called implicit discretization approach introduced in $[1,2]$. Numerical simulations are presented and demonstrate the effectiveness of the proposed discrete-time controller.
\end{abstract}

Keywords. Robust control, sliding-mode control, differential inclusion, discrete-time, trajectory tracking, Euler-Lagrange systems, finite-time convergence, Lyapunov stability, convex analysis, passivity-based controller.

\section{Introduction}

There exists a vast literature about the control of Euler-Lagrange systems, see e.g., $[12,38,39,20]$ and references therein, being the robust trajectory tracking an important problem. In the early eighties the problem of robust tracking for nonlinear robotic systems was pioneered in [34] using sliding-mode control techniques. Later in [32, 33] the methodology was improved in the context of adaptive control where the authors used a passivity-based approach rendering the closed-loop system passive and globally asymptotically stable. Set-valued control laws are common in sliding-mode control theory, where in particular the sign multifuction plays an essential role [37], but not much work has been done exploring other possibilities of multifunctions. Recently maximal monotone operators have been studied in this context $[4,10,28,36]$. The continuoustime part of the present work is a nontrivial extension of the results in $[4,32,33,34]$ and is strongly based on the theory of maximal monotone operators together with convex analysis tools. On the other hand controllers are now most of the time implemented via microprocessors, and it is of utmost importance to analyse the time-discretization of otherwise continuous-time designed algorithms. As shown in $[1,2,14,17,18,19,40,41]$, 
the time-discretization of set-valued sliding-mode control laws requires particular care, because it may yield numerical chattering (high-frequency oscillations in the output, and high-frequency bang-bang controllers) when the set-valued part of the controller is discretized in an explicit way. In fact the explicit discretization may even yield in nonlinear cases, unstable closed-loop systems [26], while the implicit method advocated in $[1,2,5,19]$ keeps the continuous-time stability properties for the systems analysed in $[11,19]$.

In this article we study fully actuated Euler-Lagrange systems and we shall focus on the robust tracking control for these systems. We start with a Euler-Lagrange system for which a desired trajectory $q_{\mathrm{d}}, \dot{q}_{\mathrm{d}}, \ddot{q}_{\mathrm{d}}$ is given and we look for a family of multivalued control law such that robust tracking is obtained, both in continuous and discrete-time. Here robustness is obtained against bounded external disturbances and also parametric uncertainties in a trajectory tracking framework, which is a big novelty with respect to $[1$, $2,4,5,19]$. In particular parametric uncertainties create an equivalent disturbance that is not uniformly upperbounded by a constant. It is also noteworthy that the results in [5] concern regulation at a fixed point, and pertain to the field of numerical analysis where a varying time-step is used, whereas the results in this paper concern feedback control design (where the sampling period is taken constant and the disturbances are unknown). The article is organised as follows. Section 2 contains mathematical preliminaries, while section 3 recalls some basic properties of Lagrange dynamics. We propose in section 4 the well-posedness analysis of the closed-loop system with set-valued controllers (existence of solutions), relaxing a stringent assumption made in [4] (see Remark 3). The stability analysis is made in section 5. In this work we do not ask for uniqueness of solutions, however we prove that all of them (if not unique) yield a tracking error with suitable stability properties. Section 6 is dedicated to the analysis of the discrete-time controller, using the implicit Euler discretization technique introduced in $[1,2,19]$ for linear timeinvariant systems with known parameters (see also [22] for a similar approach), and experimentally tested with success in $[17,18,19,40]$, in which it is shown that chattering in both, the output and the input, is almost totally suppressed. Due to the nonlinearities of the Euler-Lagrange dynamics, the design of the implicit discrete-time controller is made from a non exact discretization of the continuous plant (while the zero-order-hold discretization was used in $[1,2,19])$. The design of the discrete-time nonlinear passivitybased controller is made in section 6.1 , the stability analysis is made in section 6.2 , and convergence results as the sampling time $h>0$ goes to zero are proposed in section 6.3. Numerical simulations illustrate the theoretical developments in section 7. Conclusions end the article in section 8.

\section{Preliminaries}

In this section some preliminary results are presented together with the notation used through this article. The results from convex analysis are taken from $[8,15,16,29,30]$. Let $X$ be an $n$-dimensional real space with the classical Euclidean inner product denoted as $\langle\cdot, \cdot\rangle$ and the corresponding norm $\|\cdot\|$. A symmetric matrix $M=M^{\top}$ is called positive definite if $x^{\top} M x>0$ for any $x \in X \backslash\{0\}$. For any matrix $M \in \mathbb{R}^{n \times n}$ the norm $\|M\|_{m}$ is the induced norm given by, $\|M\|_{m}=\sup _{\|x\|=1}\|M x\|$. The unitary ball of $\mathbb{R}^{n}$ with center in 0 is denoted by $\mathbb{B}_{n}$. We denote by $\Gamma_{0}(X)$ the set of all proper, convex, and lower semicontinuous (lsc) functions from $X$ to $\mathbb{R} \cup\{+\infty\}$. The indicator of a set $C \subset X$ is the function $\Psi_{C}: X \rightarrow \mathbb{R} \cup\{+\infty\}$ which satisfies $\Psi_{C}(x)=0$ for $x \in C$ and $\Psi(x)=+\infty$ otherwise. The boundary of a set $A$ is denoted as $\operatorname{bd}(A)$.

Definition 1. Let $\Phi \in \Gamma_{0}(X)$. The subdifferential of $\Phi$ at $x$, denoted as $\partial \Phi(x)$, is the 
set-valued map given by

$$
\partial \Phi(x):=\left\{\zeta \in \mathbb{R}^{n} \mid\langle\zeta, \eta-x\rangle \leq \Phi(\eta)+\Phi(x), \forall \eta \in X\right\} .
$$

Definition 2. Let $f: X \rightarrow \mathbb{R} \cup\{+\infty\}$ be a proper function and let $\lambda>0$. The Moreau envelope of $f$ of index $\lambda$ is

$$
f^{\lambda}(x):=\inf _{w \in X}\left\{f(w)+\frac{1}{2 \lambda}\|w-x\|^{2}\right\} .
$$

Remark 1. When the function $f \in \Gamma_{0}(X)$, the Moreau envelope is known as the Moreau-Yosida approximation of $f$ of index $\lambda$ and $f^{\lambda} \in \Gamma_{0}(X)$. Furthermore, the gradient of $f^{\lambda}$ exists and is Lipschitz continuous with constant $1 / \lambda$ (see e.g., [30, Exercise 12.23]).

Definition 3. Let $f \in \Gamma_{0}(X)$ and let $x \in X$. Then the proximal map of $f$ at $x$, denoted as $\operatorname{Prox}_{f}(x)$, is the unique minimizer of $f^{1}(x)$, i.e.,

$$
f^{1}(x)=\min _{w \in X}\left\{f(w)+\frac{1}{2}\|w-x\|^{2}\right\}=f\left(\operatorname{Prox}_{f}(x)\right)+\frac{1}{2}\left\|\operatorname{Prox}_{f}(x)-x\right\|^{2} .
$$

It is important to notice that when $f=\Psi_{C}$ (the indicator of the set $C$ ), the proximal map agrees with the classical projection operator $\operatorname{Proj}_{C}(\cdot)$ given by,

$$
\operatorname{Proj}_{C}(x)=\underset{w \in C}{\arg \min } \frac{1}{2}\|w-x\|^{2} .
$$

From the previous definition it follows that

$$
f^{\lambda}(x)=f\left(\operatorname{Prox}_{\lambda f}(x)\right)+\frac{1}{2 \lambda}\left\|\operatorname{Prox}_{\lambda f}(x)-x\right\|^{2} .
$$

In words, the proximal map of $\lambda f$ at $x$ is the unique minimizer of the Moreau-Yosida approximation of $f$ of index $\lambda$. The distance between a point $w \in \mathbb{R}^{n}$ and a closed convex set $A$ is given by the expression

$$
\operatorname{dist}(x, C)=\min _{w \in C}\|x-w\|=\left\|x-\operatorname{Proj}_{C}(x)\right\| .
$$

Definition 4. Let $f: \mathbb{R}^{n} \rightarrow \mathbb{R}$ be a proper and lsc function, the conjugate function of $f$ is

$$
f^{\star}\left(x^{\star}\right)=\sup _{w \in X}\left\{\left\langle w, x^{\star}\right\rangle-f(w)\right\} .
$$

From the definition of conjugate function we have that for any two functions $f, g \in \Gamma_{0}(X)$ such that $f \geq g$, then $g^{\star} \geq f^{\star}$. The following facts will be useful in the next sections.

Lemma 1. [15, Lemma 5.2.1] Let $f \in \Gamma_{0}(X), \mathcal{A}: X \rightarrow X$ a continuous and strongly monotone operator, i.e., for any $x_{1}, x_{2} \in X$

$$
\left\langle\mathcal{A}\left(x_{1}\right)-\mathcal{A}\left(x_{2}\right), x_{1}-x_{2}\right\rangle \geq \alpha\left\|x_{1}-x_{2}\right\|,
$$

for some $\alpha>0$. Then, for each $v \in X$, there exists a unique solution $x \in X$ to the variational inequality

$$
\langle\mathcal{A} x-v, \eta-x\rangle+f(\eta)-f(x) \geq 0, \text { for all } \eta \in X .
$$

Proposition 1 (Moreau's decomposition). Let $f \in \Gamma_{0}(X)$ and $\lambda>0$. Then for any $x \in X$ we have

$$
x=\operatorname{Prox}_{\lambda f}(x)+\lambda \operatorname{Prox}_{f^{\star} / \lambda}(x / \lambda),
$$


We will use Proposition 1 in order to compute explicitly the proximal map of the norm function which will be used in Section 4. The following lemma can be found in $[8$, Example 14.5].

Lemma 2. The proximal map of index $\lambda$ of the function $f: \mathbb{R}^{n} \rightarrow \mathbb{R}_{+}, x \mapsto c\|x\|$ where $c>0$, is given by

$$
\operatorname{Prox}_{\lambda f}(x)= \begin{cases}\left(1-\frac{\lambda c}{\|x\|}\right) x, & \text { if }\|x\|>\lambda c \\ 0, & \text { if }\|x\| \leq \lambda c\end{cases}
$$

\section{Lagrangian mechanics}

Let us present the class of dynamical systems on which we will focus. We start with a nonlinear system described by its Euler-Lagrange equations in the following form

$$
M(q(t)) \ddot{q}(t)+C(q(t), \dot{q}(t)) \dot{q}(t)+G(q(t))+F(t, q(t), \dot{q}(t))=\tau(t),
$$

where $q, \dot{q}, \ddot{q} \in \mathbb{R}^{n}$ are the vectors of generalized positions, velocities and accelerations, respectively. The matrix $M(q) \in \mathbb{R}^{n \times n}$ denotes the inertia matrix of the system which satisfies $M(q)=M(q)^{\top}>0$, the term $C(q, \dot{q}) \dot{q} \in \mathbb{R}^{n}$ represents the centripetal and Coriolis forces acting on the system, the term $G(q) \in \mathbb{R}^{n}$ is the vector related with gravitational forces, the vector $F(t, q, \dot{q}) \in \mathbb{R}^{n}$ accounts for unmodeled dynamics and external disturbances. Finally, the vector $\tau \in \mathbb{R}^{n}$ represents the control input forces. The Euler-Lagrange motion equations have several useful properties. One of the most important is related to the passivity relation between the input $\tau$ and the output $\dot{q}$ [12]. On the other hand properties depending on the system in question and the definition of the matrix $C(q, \dot{q})$ through the so-called Christoffel's symbols (see, e.g., [20, Chapter 4]) hold.

Property 1. The matrices $M(q)$ and $C(q, \dot{q})$ satisfy for all differentiable functions $q$ :

$$
\frac{d}{d t} M(q(t))=C(q(t), \dot{q}(t))+C^{\top}(q(t), \dot{q}(t))
$$

Notice that the previous property implies that $\dot{M}(q)-2 C(q, \dot{q})$ is skew-symmetric. The following assumptions are almost standard [20, 12].

Assumption 1. The matrices $M(q), C(q, \dot{q})$ together with the vectors $G(q)$ and $F(t, q, \dot{q})$ satisfy the following inequalities for all $(t, q, \dot{q}) \in \mathbb{R}_{+} \times \mathbb{R}^{n} \times \mathbb{R}^{n}$ and some positive bounded constants $k_{1}, k_{2}, k_{C}, k_{G}$ and $k_{F}$ :

$$
\begin{aligned}
0<k_{1} \leq\|M(q)\|_{m} & \leq k_{2}, \\
\|C(q, \dot{q})\|_{m} & \leq k_{C}\|\dot{q}\|, \\
\|G(q)\| & \leq k_{G}\|q\|, \\
\|F(t, q, \dot{q})\| & \leq k_{F} .
\end{aligned}
$$

Assumption 2. There exists a constant $k_{3}$ such that for all $x, y, z \in \mathbb{R}^{n}$

$$
\|M(x) z-M(y) z\| \leq k_{3}\|x-y\|\|z\| .
$$

Assumption 3. The function $h: \mathbb{R}^{n} \times \mathbb{R}^{n} \rightarrow \mathbb{R}^{n}$ defined by $h\left(x_{1}, x_{2}, x_{3}\right):=C\left(x_{1}, x_{2}\right) x_{3}$ is locally Lipschitz.

Assumption 4. The function $F\left(t, x_{1}, x_{2}\right)$ is continuous in $t$, uniformly locally Lipschitz in $\left(x_{1}, x_{2}\right)$. 
Assumption 5. The function $G(\cdot)$ is Lipschitz continuous and satisfies $0=G(0) \leq$ $G(x)$ for all $x \in \mathbb{R}^{n}$.

All previous assumptions will be used in the following section. Our control objective is described as follows:

Problem 1. Given the nonlinear system (8), design a control law $\tau(\cdot)$ such that the tracking of a desired trajectory $\left(q_{\mathrm{d}}, \dot{q}_{\mathrm{d}}, \ddot{q}_{\mathrm{d}}\right)$ - considered bounded and with bounded first and second derivatives - is achieved in the presence of the disturbance $F(t, q, \dot{q})$ and parametric uncertainties in $M(q), C(q, \dot{q})$ and $G(q)$.

\section{Well-posedness of the continuous-time closed-loop dy- namics}

\subsection{Multivalued control law}

In this section we start setting the multivalued control law based on the fixed-parameter controller proposed in $[32,33]$. Subsequently we will establish the existence of solutions and stability of the closed-loop. Let us introduce the position error $\tilde{q}=q-q_{\mathrm{d}}$ and the sliding surface $\sigma=\dot{\tilde{q}}+\Lambda \tilde{q}$ which will be used in order to maintain the error signal around zero, where the matrix $-\Lambda \in \mathbb{R}^{n \times n}$ is Hurwitz and satisfies $K_{p} \Lambda=\Lambda^{\top} K_{p}>0$ for a symmetric and positive definite matrix $K_{p} \in \mathbb{R}^{n \times n}$. The proposed control law has the following form

$$
\tau(q, \dot{q})=\hat{M}(q) \ddot{q}_{\mathrm{r}}+\hat{C}(q, \dot{q}) \dot{q}_{\mathrm{r}}+\hat{G}(q)-K_{p} \tilde{q}+u,
$$

where $\dot{q}_{\mathrm{r}}=\dot{q}_{\mathrm{d}}-\Lambda \tilde{q}, K_{p} \in \mathbb{R}^{n \times n}, K_{p}=K_{p}^{\top}>0$, the term $u$ is related to the multivalued part of the controller and is specified below. Moreover, the matrices $\hat{M}(q), \hat{C}(q, \dot{q})$ and $\hat{G}(q)$ represent the nominal system available for control and it is assumed that all of them fulfill Assumptions 1 to 5 (although with different bounds) i.e., we assume that all the uncertainties are in the system parameters and not in the structure of the matrices.

Assumption 6. The matrices $\hat{M}(q), \hat{C}(q, \dot{q})$ together with the vector $\hat{G}(q)$ satisfy the following inequalities for all $(t, q, \dot{q}) \in \mathbb{R}_{+} \times \mathbb{R}^{n} \times \mathbb{R}^{n}$ and some positive bounded constants $\hat{k}_{1}, \hat{k}_{2}, \hat{k}_{C}$ and $\hat{k}_{G}$

$$
\begin{aligned}
0<\hat{k}_{1} \leq\|\hat{M}(q)\|_{m} & \leq \hat{k}_{2}, \\
\|\hat{C}(q, \dot{q})\|_{m} & \leq \hat{k}_{C}\|\dot{q}\|, \\
\|\hat{G}(q)\| & \leq \hat{k}_{G}\|q\| .
\end{aligned}
$$

After some simple manipulations, the closed-loop system results in

$$
\left\{\begin{array}{l}
M(q) \dot{\sigma}+C(q, \dot{q}) \sigma+K_{p} \tilde{q}+\xi(t, \sigma, \tilde{q})=u, \\
\dot{\tilde{q}}=\sigma-\Lambda \tilde{q},
\end{array}\right.
$$

where the new function $\xi: \mathbb{R}_{+} \times \mathbb{R}^{n} \times \mathbb{R}^{n} \rightarrow \mathbb{R}^{n}$ accounts for all the uncertainties in the system and is given by

$$
\xi(t, \sigma, \tilde{q})=F(t, q, \dot{q})+\Delta M(q) \ddot{q}_{\mathrm{r}}+\Delta C(q, \dot{q}) \dot{q}_{\mathrm{r}}+\Delta G(q),
$$

where $\Delta M(q)=M(q)-\hat{M}(q), \Delta C(q, \dot{q})=C(q, \dot{q})-\hat{C}(q, \dot{q})$ and $\Delta G(q)=G(q)-\hat{G}(q)$. Notice that the closed-loop system (10) slightly differs from the closed-loop system in $[32,33]$, since we have omitted the term proportional to $\sigma$ and we have added the term 
$K_{p} \tilde{q}$ instead. Additionally, it is worth to mention that the function $\xi$ is not uniformly bounded but it is still upper-bounded by a locally Lipschitz continuous function of positions and velocities, as the following proposition reveals. This fact is a nice feature of the passivity-based control, not shared by other nonlinear control techniques like feedback linearization.

Proposition 2. The function $\xi(t, \sigma, \tilde{q})$ satisfies

$$
\|\xi(t, \sigma, \tilde{q})\| \leq \beta(\sigma, \tilde{q}),
$$

where $\beta(\sigma, \tilde{q})=c_{1}+c_{2}\|\sigma\|+c_{3}\|\tilde{q}\|+c_{4}\|\tilde{q}\|\|\sigma\|+c_{5}\|\tilde{q}\|^{2}$, for bounded positive constants $c_{i}, i=1, \ldots, 5$.

Proof. From (11) we have

$$
\left.\|\xi(t, \sigma, \tilde{q})\| \leq\|F(t, q, \dot{q})\|+\left\|\Delta M(q) \ddot{q}_{\mathrm{r}}\right\|+\| \Delta C(q, \dot{q}) \dot{q}_{\mathrm{r}}\right)\|+\| \Delta G(q) \| .
$$

From Assumption 1 it follows that the first term on the right-hand side of (12) is bounded by a constant $k_{F}$. The next term satisfies

$$
\begin{aligned}
\left\|\Delta M(q) \ddot{q}_{\mathrm{r}}\right\| & =\left\|(M(q)-\hat{M}(q)) \ddot{q}_{\mathrm{r}}\right\| \leq\left(k_{2}+\hat{k}_{2}\right)\left\|\ddot{q}_{\mathrm{r}}\right\|, \\
& \leq\left(k_{2}+\hat{k}_{2}\right)\left(\left\|\ddot{q}_{\mathrm{d}}\right\|+\|\Lambda\|_{m}\|\dot{\tilde{q}}\|\right) \\
& \leq\left(k_{2}+\hat{k}_{2}\right)\left(\left\|\ddot{q}_{\mathrm{d}}\right\|+\|\Lambda\|_{m}\|\sigma\|+\|\Lambda\|_{m}^{2}\|\tilde{q}\|\right) .
\end{aligned}
$$

Following the same procedure for the term $\left\|\Delta C(q, \dot{q}) \dot{q}_{\mathrm{r}}\right\|$ we obtain

$$
\begin{aligned}
\left\|\Delta C(q, \dot{q}) \dot{q}_{\mathrm{r}}\right\| & =\left\|(C(q, \dot{q})-\hat{C}(q, \dot{q})) \dot{q}_{\mathrm{r}}\right\| \leq\left(k_{C}+\hat{k}_{C}\right)\|\dot{q}\|\left\|\dot{q}_{\mathrm{r}}\right\| \\
& \leq\left(k_{C}+\hat{k}_{C}\right)\left(\left\|\dot{q}_{\mathrm{d}}\right\|+\|\sigma-\Lambda \tilde{q}\|\right)\left(\left\|\dot{q}_{\mathrm{d}}-\Lambda \tilde{q}\right\|\right) \\
& \leq\left(k_{C}+\hat{k}_{C}\right)\left(\left\|\dot{q}_{\mathrm{d}}\right\|+\|\sigma\|+\|\Lambda\|_{m}\|\tilde{q}\|\right)\left(\left\|\dot{q}_{\mathrm{d}}\right\|+\|\Lambda\|_{m}\|\tilde{q}\|\right) \\
& =\left(k_{C}+\hat{k}_{C}\right)\left[\left(\left\|\dot{q}_{\mathrm{d}}\right\|+\|\Lambda\|_{m}\|\tilde{q}\|\right)^{2}+\left(\left\|\dot{q}_{\mathrm{d}}\right\|+\|\Lambda\|_{m}\|\tilde{q}\|\right)\|\sigma\|\right] .
\end{aligned}
$$

Finally,

$$
\begin{aligned}
\|\Delta G(q)\| & =\|G(q)-\hat{G}(q)\| \leq\left(k_{G}+\hat{k}_{G}\right)\|q\| \\
& \leq\left(k_{G}+\hat{k}_{G}\right)\left(\left\|q_{d}\right\|+\|\tilde{q}\|\right) .
\end{aligned}
$$

Inasmuch as the variables $q_{\mathrm{d}}, \dot{q}_{\mathrm{d}}$ and $\ddot{q}_{\mathrm{d}}$ are known and bounded, we obtain the desired result with

$$
\begin{aligned}
& c_{1}:=k_{F}+\left(k_{2}+\hat{k}_{2}\right)\left\|\ddot{q}_{\mathrm{d}}\right\|+\left(k_{C}+\hat{k}_{C}\right)\left\|\dot{q}_{\mathrm{d}}\right\|^{2}+\left(k_{G}+\hat{k}_{G}\right)\left\|q_{\mathrm{d}}\right\|, \\
& c_{2}:=\left(k_{2}+\hat{k}_{2}\right)\|\Lambda\|_{m}+\left(k_{C}+\hat{k}_{C}\right)\left\|\dot{q}_{\mathrm{d}}\right\|, \\
& c_{3}:=\left(k_{2}+\hat{k}_{2}\right)\|\Lambda\|_{m}^{2}+2\left(k_{C}+\hat{k}_{C}\right)\left\|\dot{q}_{\mathrm{d}}\right\|+k_{G}+\hat{k}_{G}, \\
& c_{4}:=\left(k_{C}+\hat{k}_{C}\right)\|\Lambda\|_{m}, \\
& c_{5}:=\left(k_{C}+\hat{k}_{C}\right)\|\Lambda\|_{m}^{2} .
\end{aligned}
$$

Remark 2. Notice that in the case when we have no parametric uncertainty, the disturbance $\xi$ is uniformly bounded by a constant $k_{F}>0$ (see Assumption 1). 
The importance of the control law (9) relies on the fact that it imposes (in the closedloop system) a new passivity relation between the new input $u \in \mathbb{R}^{n}$ and the output $\sigma \in \mathbb{R}^{n}[12$, Chapter 7]. Now we define the multivalued part of the control law $\tau$ as,

$$
-u \in \gamma(\sigma, \tilde{q}) \partial \Phi(\sigma),
$$

where the function $\gamma: \mathbb{R}^{n} \times \mathbb{R}^{n} \rightarrow \mathbb{R}_{+}$is locally Lipschitz continuous and is specified below in Lemma 3. Additionally, $\Phi \in \Gamma_{0}\left(\mathbb{R}^{n}\right)$ and it is selected in such a way that the following assumption is fulfilled.

Assumption 7. The function $\Phi \in \Gamma_{0}\left(\mathbb{R}^{n}\right)$ satisfies $0=\Phi(0) \leq \Phi(w)$ for all $w \in \mathbb{R}^{n}$. Moreover, we have that $0 \in \operatorname{int} \partial \Phi(0)$.

Proposition 3. The following assertions are equivalent:

- $0 \in \operatorname{int} \partial \Phi(0)$,

- There exists $\alpha>0$ such that, $\Phi(\cdot) \geq \alpha\|\cdot\|$.

Proof. Let $0 \in \operatorname{int} \partial \Phi(0)$, i.e., there exists $\varepsilon>0$ such that for all $\rho \in \varepsilon \mathbb{B}_{n}$ and all $\eta \in \mathbb{R}^{n}$, we have $\langle\rho, \eta\rangle \leq \Phi(\eta)$. Equivalently, $\sup \left\{\langle\rho, \eta\rangle \mid \rho \in \varepsilon \mathbb{B}_{n}\right\} \leq \Phi(\eta)$ for all $\eta \in \mathbb{R}^{n}$ and consequently $\varepsilon\|\eta\| \leq \Phi(\eta)$ for all $\eta \in \mathbb{R}^{n}$.

\subsection{Existence of solutions}

The next step consists in establishing the existence of solutions for the closed-loop system (10) when the multivalued control (13) is applied. To this end we start using the Yosida approximation of the set-valued map $\partial \Phi(\cdot)$ and after proving the boundeness of the variables $\sigma$ and $\tilde{q}$, we can conclude the existence of solutions of the differential equation taking a limit process. Actually, let $\Phi^{\lambda}(\sigma)$ be the Moreau-Yosida approximation of $\Phi$ of index $\lambda$ at the point $\sigma \in \mathbb{R}^{n}$. So, as is pointed out in [6, Th. 4 Sec. 3.4], the gradient $\nabla \Phi^{\lambda}(\sigma)$ corresponds to the Yosida approximation of $\partial \Phi(\sigma)$ such that the approximated closed-loop dynamics satisfies

$$
\left\{\begin{array}{l}
M\left(q_{\lambda}\right) \dot{\sigma}_{\lambda}+C\left(q_{\lambda}, \dot{q}_{\lambda}\right) \sigma_{\lambda}+K_{p} \tilde{q}_{\lambda}+\xi\left(t, \sigma_{\lambda}, \tilde{q}_{\lambda}\right)=-\gamma\left(\sigma_{\lambda}, \tilde{q}_{\lambda}\right) \nabla \Phi^{\lambda}\left(\sigma_{\lambda}\right), \\
\dot{\tilde{q}}_{\lambda}=\sigma_{\lambda}-\Lambda \tilde{q}_{\lambda} .
\end{array}\right.
$$

As a first step we will prove that closed-loop system (14) is well-posed.

Lemma 3. Let Assumptions 1-7 hold. Then the system (14) has a unique bounded solution $\left(\sigma_{\lambda}, \tilde{q}_{\lambda}\right)$ for any $\lambda>0$, initial data $\left(\sigma_{0}, \tilde{q}_{0}\right)$ and $t \geq 0$ whenever

$$
\frac{\alpha}{2} \gamma\left(\sigma_{\lambda}, \tilde{q}_{\lambda}\right) \geq \beta\left(\sigma_{\lambda}, \tilde{q}_{\lambda}\right), \text { for all }\left\|\sigma_{\lambda}\right\|>\lambda \alpha,
$$

where the function $\beta$ is specified in Proposition 2 and $\alpha$ is given in Proposition 3. Moreover, the system is globally practically asymptotically stable.

Proof. Clearly (14) is equivalent to

$$
\left[\begin{array}{c}
\dot{\sigma}_{\lambda} \\
\dot{\tilde{q}} \lambda
\end{array}\right]=\left[\begin{array}{c}
-M^{-1}\left(q_{\lambda}\right)\left(C\left(q_{\lambda}, \dot{q}_{\lambda}\right) \sigma_{\lambda}+K_{p} \tilde{q}_{\lambda}+\xi\left(t, \sigma_{\lambda}, \tilde{q}_{\lambda}\right)+\gamma\left(\sigma_{\lambda}, \tilde{q}_{\lambda}\right) \nabla \Phi^{\lambda}\left(\sigma_{\lambda}\right)\right) \\
\sigma_{\lambda}-\Lambda \tilde{q}_{\lambda}
\end{array}\right] .
$$

Since all the terms in $\xi\left(t, \sigma_{\lambda}, \tilde{q}_{\lambda}\right)$ are locally Lipschitz together with the function $\gamma$ and the gradient $\nabla \Phi^{\lambda}$, it is easy to see that the term $g\left(\sigma_{\lambda}, \tilde{q}_{\lambda}\right):=C\left(q_{\lambda}, \dot{q}_{\lambda}\right) \sigma_{\lambda}+K_{p} \tilde{q}_{\lambda}+$ $\xi\left(t, \sigma_{\lambda}, \tilde{q}_{\lambda}\right)+\gamma\left(\sigma_{\lambda}, \tilde{q}_{\lambda}\right) \nabla \Phi^{\lambda}\left(\sigma_{\lambda}\right)$ is locally Lipschitz too. So, due to Assumption 2 it is possible to prove that $M^{-1}\left(q_{\lambda}\right) g\left(\sigma_{\lambda}, \tilde{q}_{\lambda}\right)$ is locally Lipschitz continuous. In conclusion, 
the right-hand side of (16) is locally Lipschitz continuous, and therefore there exists $T>0$ such that a unique solution in the Caratheodory's sense exists in the interval $[0, T)$. Now we prove that the solution exists for all $T>0$. Following [35] and [12, p. 403] let us consider the energy function, $H\left(\sigma_{\lambda}, \tilde{q}_{\lambda}\right)=\frac{1}{2} \sigma_{\lambda}^{\top} M\left(q_{\lambda}\right) \sigma_{\lambda}+\frac{1}{2} \tilde{q}_{\lambda}^{\top} K_{p} \tilde{q}_{\lambda}$. The derivative of $H$ along trajectories of (16) takes the form

$$
\begin{aligned}
\dot{H}\left(\sigma_{\lambda}, \tilde{q}_{\lambda}\right)= & \frac{1}{2} \sigma_{\lambda}^{\top} \dot{M}\left(q_{\lambda}\right) \sigma_{\lambda}+\sigma_{\lambda}^{\top} M\left(q_{\lambda}\right) \dot{\sigma}_{\lambda}+\tilde{q}_{\lambda}^{\top} K_{p} \dot{\tilde{q}}_{\lambda} \\
= & \frac{1}{2} \sigma_{\lambda}^{\top}\left(\dot{M}\left(q_{\lambda}\right)-2 C\left(q_{\lambda}, \dot{q}_{\lambda}\right)\right) \sigma_{\lambda} \\
& -\sigma_{\lambda}^{\top}\left(K_{p} \tilde{q}_{\lambda}+\xi\left(t, \sigma_{\lambda}, \tilde{q}_{\lambda}\right)+\gamma\left(\sigma_{\lambda}, \tilde{q}_{\lambda}\right) \nabla \Phi^{\lambda}\left(\sigma_{\lambda}\right)\right)+\tilde{q}_{\lambda}^{\top} K_{p}\left(\sigma_{\lambda}-\Lambda \tilde{q}_{\lambda}\right) \\
= & -\tilde{q}_{\lambda}^{\top} K_{p} \Lambda \tilde{q}_{\lambda}-\gamma\left(\sigma_{\lambda}, \tilde{q}_{\lambda}\right)\left\langle\nabla \Phi^{\lambda}\left(\sigma_{\lambda}\right), \sigma_{\lambda}\right\rangle-\left\langle\xi\left(t, \sigma_{\lambda}, \tilde{q}_{\lambda}\right), \sigma_{\lambda}\right\rangle .
\end{aligned}
$$

The following step consists in describing more precisely the term $\left\langle\nabla \Phi^{\lambda}\left(\sigma_{\lambda}\right), \sigma_{\lambda}\right\rangle$. As was pointed out in Remark 1, the function $\Phi^{\lambda}$ is convex (and differentiable), therefore its gradient satisfies $\left\langle\nabla \Phi^{\lambda}\left(\sigma_{\lambda}\right), \eta-\sigma_{\lambda}\right\rangle \leq \Phi^{\lambda}(\eta)-\Phi^{\lambda}\left(\sigma_{\lambda}\right)$ for all $\eta \in \mathbb{R}^{n}$ [16, Th. 4.1.1]. In particular $-\left\langle\nabla \Phi^{\lambda}\left(\sigma_{\lambda}\right), \sigma_{\lambda}\right\rangle \leq-\Phi^{\lambda}\left(\sigma_{\lambda}\right) \leq-f^{\lambda}\left(\sigma_{\lambda}\right)$ where we have used Proposition 3 with $f(\cdot)=\alpha\|\cdot\|$. Moreover, using (5) it follows that

$$
-\left\langle\nabla \Phi^{\lambda}\left(\sigma_{\lambda}\right), \sigma_{\lambda}\right\rangle \leq-f^{\lambda}\left(\sigma_{\lambda}\right)=-\alpha\left\|\operatorname{Prox}_{\lambda f}\left(\sigma_{\lambda}\right)\right\|-\frac{1}{2 \lambda}\left\|\operatorname{Prox}_{\lambda f}\left(\sigma_{\lambda}\right)-\sigma_{\lambda}\right\|^{2} .
$$

Assume first that $\left\|\sigma_{\lambda}\right\| \leq \alpha \lambda$. The substitution of (18) into (17) together with (7) in Lemma 2 leads to,

$$
\begin{aligned}
\dot{H}\left(\sigma_{\lambda}, \tilde{q}_{\lambda}\right) \leq & -\tilde{q}_{\lambda}^{\top} K_{p} \Lambda \tilde{q}_{\lambda}-\gamma\left(\sigma_{\lambda}, \tilde{q}_{\lambda}\right)\left(\alpha\left\|\operatorname{Prox}_{\lambda f}\left(\sigma_{\lambda}\right)\right\|+\frac{1}{2 \lambda}\left\|\operatorname{Prox}_{\lambda f}\left(\sigma_{\lambda}\right)-\sigma_{\lambda}\right\|^{2}\right) \\
& -\left\langle\xi\left(t, \sigma_{\lambda}, \tilde{q}_{\lambda}\right), \sigma_{\lambda}\right\rangle \\
\leq & -\tilde{q}_{\lambda}^{\top} K_{p} \Lambda \tilde{q}_{\lambda}-\frac{1}{2 \lambda} \gamma\left(\sigma_{\lambda}, \tilde{q}_{\lambda}\right)\left\|\sigma_{\lambda}\right\|^{2}-\left\langle\xi\left(t, \sigma_{\lambda}, \tilde{q}_{\lambda}\right), \sigma_{\lambda}\right\rangle \\
\leq & -\tilde{q}_{\lambda}^{\top} K_{p} \Lambda \tilde{q}_{\lambda}-\left(\frac{1}{2 \lambda} \gamma\left(\sigma_{\lambda}, \tilde{q}_{\lambda}\right)\left\|\sigma_{\lambda}\right\|-\left\|\xi\left(t, \sigma_{\lambda}, \tilde{q}_{\lambda}\right)\right\|\right)\left\|\sigma_{\lambda}\right\| \\
\leq & -\tilde{q}_{\lambda}^{\top} K_{p} \Lambda \tilde{q}_{\lambda}-\left(\frac{1}{2 \lambda} \gamma\left(\sigma_{\lambda}, \tilde{q}_{\lambda}\right)\left\|\sigma_{\lambda}\right\|-\beta\left(\sigma_{\lambda}, \tilde{q}_{\lambda}\right)\right)\left\|\sigma_{\lambda}\right\| .
\end{aligned}
$$

On the other hand, when $\left\|\sigma_{\lambda}\right\|>\lambda \alpha$ we have (still relying on (7) and (17)):

$$
\begin{aligned}
\dot{H}\left(\sigma_{\lambda}, \tilde{q}_{\lambda}\right) \leq & -\tilde{q}_{\lambda}^{\top} K_{p} \Lambda \tilde{q}_{\lambda}-\gamma\left(\sigma_{\lambda}, \tilde{q}_{\lambda}\right)\left[\alpha\left(1-\frac{\lambda \alpha}{\left\|\sigma_{\lambda}\right\|}\right)\left\|\sigma_{\lambda}\right\|+\right. \\
& \left.\frac{1}{2 \lambda}\left\|\sigma_{\lambda}-\left(1-\frac{\lambda \alpha}{\left\|\sigma_{\lambda}\right\|}\right) \sigma_{\lambda}\right\|^{2}\right]+\left\|\xi\left(t, \sigma_{\lambda}, \tilde{q}_{\lambda}\right)\right\|\left\|\sigma_{\lambda}\right\| \\
= & -\tilde{q}_{\lambda}^{\top} K_{p} \Lambda \tilde{q}_{\lambda}-\left[\alpha\left(1-\frac{\lambda \alpha}{2\left\|\sigma_{\lambda}\right\|}\right) \gamma\left(\sigma_{\lambda}, \tilde{q}_{\lambda}\right)-\left\|\xi\left(t, \sigma_{\lambda}, \tilde{q}_{\lambda}\right)\right\|\right]\left\|\sigma_{\lambda}\right\| .
\end{aligned}
$$

Now, since we are in the case $\left\|\sigma_{\lambda}\right\|>\lambda \alpha$ we have that,

$$
1-\frac{\lambda \alpha}{2\left\|\sigma_{\lambda}\right\|} \geq \inf _{\left\|\sigma_{\lambda}\right\|>\lambda \alpha}\left\{1-\frac{\lambda \alpha}{2\left\|\sigma_{\lambda}\right\|}\right\}=1-\sup _{\left\|\sigma_{\lambda}\right\|>\lambda \alpha}\left\{\frac{\lambda \alpha}{2\left\|\sigma_{\lambda}\right\|}\right\}=1-\frac{1}{2}=\frac{1}{2} .
$$

Hence,

$$
\dot{H}\left(\sigma_{\lambda}, \tilde{q}_{\lambda}\right) \leq-\tilde{q}_{\lambda}^{\top} K_{p} \Lambda \tilde{q}_{\lambda}-\left(\frac{\alpha}{2} \gamma\left(\sigma_{\lambda}, \tilde{q}_{\lambda}\right)-\beta\left(\sigma_{\lambda}, \tilde{q}_{\lambda}\right)\right)\left\|\sigma_{\lambda}\right\|
$$


It becomes clear from (19) and (20), that if we want $H$ to decrease on all $\mathbb{R}^{n}$ then we must have

$$
\gamma\left(\sigma_{\lambda}, \tilde{q}_{\lambda}\right)\left\|\sigma_{\lambda}\right\| \geq 2 \lambda \beta\left(\sigma_{\lambda}, \tilde{q}_{\lambda}\right), \text { for all }\left\|\sigma_{\lambda}\right\| \leq \lambda \alpha,
$$

and,

$$
\frac{\alpha}{2} \gamma\left(\sigma_{\lambda}, \tilde{q}_{\lambda}\right) \geq \beta\left(\sigma_{\lambda}, \tilde{q}_{\lambda}\right), \text { for all }\left\|\sigma_{\lambda}\right\|>\lambda \alpha .
$$

The first of the inequalities seems to cause some difficulties since it depends explicitly on the term $\left\|\sigma_{\lambda}\right\|$, which in this case is considered smaller than $\lambda \alpha$. In fact as $\left\|\sigma_{\lambda}\right\|$ decreases, the term $\gamma\left(\sigma_{\lambda}, \tilde{q}_{\lambda}\right)$ should increase in order to satisfy the inequality. For this reason we are going to focus on the second inequality, which if it is satisfied, implies practical stability. Indeed, assuming that $\alpha \gamma\left(\sigma_{\lambda}, \tilde{q}_{\lambda}\right) \geq 2 \beta\left(\sigma_{\lambda}, \tilde{q}_{\lambda}\right)+\delta$, for some $\delta>0$ and for all $\left\|\sigma_{\lambda}\right\|>\alpha \lambda$, leads us to $\dot{H}\left(\sigma_{\lambda}, \tilde{q}_{\lambda}\right)<0$ which implies that the state variable $\sigma_{\lambda}$ will converge to the ball $\alpha \lambda \mathbb{B}_{n}$, i.e., $\sup _{t \in \mathbb{R}_{+}}\left\|\sigma_{\lambda}(t)\right\| \leq \bar{\sigma}$, for some $\bar{\sigma}<+\infty$. On the other hand we have from (16) that

$$
\begin{aligned}
\left\|\tilde{q}_{\lambda}(t)\right\| & =\left\|e^{-\Lambda t} \tilde{q}_{0}+\int_{0}^{t} e^{-\Lambda(t-s)} \sigma_{\lambda}(s) d s\right\| \\
& \leq\left\|e^{-\Lambda t} \tilde{q}_{0}\right\|+\bar{\sigma} \int_{0}^{t}\left\|e^{-\Lambda \theta}\right\| d \theta \\
& \leq\left\|e^{-\Lambda t} \tilde{q}_{0}\right\|+\bar{\sigma} p,
\end{aligned}
$$

for some $p<+\infty$ and we have used the change of variables $t-s=\theta$ together with the fact that the term $\left\|e^{-\Lambda t}\right\| \in \mathcal{L}_{1}([0,+\infty), \mathbb{R})$, (see, e.g., the proof of Theorem 6.10 in [31]). Hence, it becomes clear that the pair $\left(\sigma_{\lambda}, \tilde{q}_{\lambda}\right)$ is ultimately bounded (see e.g., [21, Sec. 4.8]) and boundedness of solutions of (14) over all $t \in \mathbb{R}_{+}$follows.

Remark 3. Notice that the proof of the previous Lemma significantly differs from the proof of Lemma 4.1 in [4], because we relax the stringent Assumption $\mathcal{H}_{\Phi, F}$ in [4] which overly constrains the relation between the Moreau-Yosida approximations of $\Phi(\cdot)$ and the disturbance. We used only the property $0 \in \operatorname{int} \partial \Phi(0)$ of the design function $\Phi$ and (15) instead (see Assumption 7), which allows us to consider a much larger and realistic class of disturbances.

Once the well-posedness of the approximated system has been established, we can obtain the existence of solutions for the differential inclusion (10)-(13) applying [4, Theorem 4.2 ], which we repeat here only for completeness.

Theorem 1. [4, Theorem 4.1] Let Assumptions 1-7 hold. Then for every $\left(\sigma_{0}, \tilde{q}_{0}\right) \in$ $\mathbb{R}^{n} \times \mathbb{R}^{n}$, there exists a solution $\sigma:[0,+\infty) \rightarrow \mathbb{R}^{n}, \tilde{q}:[0,+\infty) \rightarrow \mathbb{R}^{n}$ of $(10)-(13)$ in the following sense:

- $\sigma$ is continuous with derivative $\dot{\sigma}$ continuous and bounded in bounded sets.

- $\tilde{q}$ is continuous with derivative $\dot{\tilde{q}}$ continuous and bounded in bounded sets.

- (10)-(13) is satisfied for a.e. $t \in[0,+\infty)$

- $\sigma(0)=\sigma_{0}$ and $\tilde{q}(0)=\tilde{q}_{0}$.

The proof of Theorem 1 is detailed in [4], taking into account that the term $\ddot{q}$ in [4] refers to $\dot{\sigma}$ in our setting.

Remark 4. It is worth to mention that Theorem 1 does not guarantee uniqueness of solutions. The uniqueness property can be achieved asking for more stringent properties which are rarely satisfied in practical cases, see [4, Section 5]. 


\subsection{Case with a constant gain $\gamma$}

The well-posedness of the closed-loop system (10)-(13) has been proved above making use of a state dependent gain $\gamma(\sigma, \tilde{q})$ which satisfies (15). An important case of study is when the control gain is set constant, which simplifies the implementation. Due to the state dependency of the disturbance $\xi$, it becomes clear that we cannot obtain the same stability properties for the new closed-loop system as before, but the existence of solutions is still assured, as the following Lemma shows.

Lemma 4. Let Assumptions 1-7 hold. Consider the following system

$$
\left\{\begin{array}{l}
M\left(q_{\lambda}\right) \dot{\sigma}_{\lambda}+C\left(q_{\lambda}, \dot{q}_{\lambda}\right) \sigma_{\lambda}+K_{p} \tilde{q}_{\lambda}+\xi\left(t, \sigma_{\lambda}, \tilde{q}_{\lambda}\right)=-\gamma \nabla \Phi^{\lambda}\left(\sigma_{\lambda}\right), \\
\dot{\tilde{q}}_{\lambda}=\sigma_{\lambda}-\Lambda \tilde{q}_{\lambda},
\end{array}\right.
$$

where $\gamma$ is a positive constant, and consider the compact set,

$$
W_{R, K_{p}}:=\left\{\left(\sigma_{\lambda}, \tilde{q}_{\lambda}\right) \in \mathbb{R}^{n} \times \mathbb{R}^{n} \mid H\left(\sigma_{\lambda}, \tilde{q}_{\lambda}\right) \leq R\right\},
$$

where

$$
H\left(\sigma_{\lambda}, \tilde{q}_{\lambda}\right)=\frac{1}{2} \sigma_{\lambda}^{\top} M\left(q_{\lambda}\right) \sigma_{\lambda}+\frac{1}{2} \tilde{q}_{\lambda}^{\top} K_{p} \tilde{q}_{\lambda} .
$$

Then for any initial condition $\left(\sigma_{\lambda}(0), \tilde{q}_{\lambda}(0)\right)$ which lies in $W_{R, K_{p}}$ and any $R>0$ fixed, the unique solution of (21) is bounded for all $\lambda>0$ such that $\lambda^{2} \leq 2 R /\left(\alpha^{2} \max \left\{k_{2}, k_{p_{2}}\right\}\right)$, where $k_{p_{2}}$ is the maximum eigenvalue of $K_{p}$, and all $t \geq 0$ whenever $\gamma>2 R_{\xi} / \alpha$, where $R_{\xi}>0$ is a constant that satisfies

$$
R_{\xi}=\max _{(\sigma, \tilde{q}) \in W_{R, K_{p}}} \beta(\sigma, \tilde{q}) .
$$

In other words the system is semi-globally practically asymptotically stable.

Proof. The proof is similar to the proof of Lemma 3. First the positive invariance of $W_{R, K_{p}}$ is shown by proving that $\dot{H}<0$ for all $\left(\sigma_{\lambda}, \tilde{q}_{\lambda}\right) \in \operatorname{bd}\left(W_{R, K_{p}}\right)$. Indeed, the condition $\lambda^{2} \leq 2 R /\left(\alpha^{2} \max \left\{k_{2}, k_{p_{2}}\right\}\right)$ implies that for all $\left(\sigma_{\lambda}, \tilde{q}_{\lambda}\right) \in \operatorname{bd}\left(W_{R, K_{p}}\right)$ we have $\left\|\sigma_{\lambda}\right\| \geq \alpha \lambda$. Namely, let $k_{\max }=\max \left\{k_{2}, k_{p 2}\right\} / 2$, since $\alpha^{2} \lambda^{2} \leq R / k_{\max }$ we have that $\alpha \lambda \mathbb{B}_{2 n} \subseteq \sqrt{R / k_{\max }} \mathbb{B}_{2 n}$. Hence for any $\left(\sigma_{\lambda}, \tilde{q}_{\lambda}\right) \in \alpha \lambda \mathbb{B}_{2 n}$ it follows that

$$
\begin{aligned}
R & \geq k_{\max } \lambda^{2} \alpha^{2} \geq k_{\max }\left(\left\|\sigma_{\lambda}\right\|^{2}+\left\|\tilde{q}_{\lambda}\right\|^{2}\right) \geq \frac{1}{2} k_{2}\left\|\sigma_{\lambda}\right\|^{2}+\frac{1}{2} k_{p_{2}}\|\tilde{q}\|^{2} \\
& \geq \frac{1}{2} \sigma_{\lambda}^{\top} M\left(q_{\lambda}\right) \sigma_{\lambda}+\frac{1}{2} \tilde{q}_{\lambda}^{\top} K_{p} \tilde{q}_{\lambda} .
\end{aligned}
$$

Therefore $\alpha \lambda \mathbb{B}_{2 n} \subseteq W_{R, K_{p}}$ and it follows that $\left\|\sigma_{\lambda}\right\| \geq \alpha \lambda$ in the boundary of $W_{R, K_{p}}$. On the other hand $\dot{H}$ satisfies

$$
\begin{aligned}
\dot{H} & =-\tilde{q}_{\lambda}^{\top} K_{p} \Lambda \tilde{q}_{\lambda}-\gamma\left\langle\nabla \Phi^{\lambda}\left(\sigma_{\lambda}\right), \sigma_{\lambda}\right\rangle-\left\langle\xi\left(t, \sigma_{\lambda}, \tilde{q}_{\lambda}\right), \sigma_{\lambda}\right\rangle \\
& \leq-\tilde{q}_{\lambda}^{\top} K_{p} \Lambda \tilde{q}_{\lambda}-\gamma f^{\lambda}\left(\sigma_{\lambda}\right)+\beta\left(\sigma_{\lambda}, \tilde{q}_{\lambda}\right)\left\|\sigma_{\lambda}\right\|,
\end{aligned}
$$

where we made use of Propositions 2 and 3, the fact that a differentiable convex function always satisfies $\left\langle\nabla \Phi^{\lambda}\left(\sigma_{\lambda}\right), \eta-\sigma_{\lambda}\right\rangle \leq \Phi^{\lambda}(\eta)-\Phi^{\lambda}\left(\sigma_{\lambda}\right)$, for all $\eta \in \mathbb{R}^{n}$ and recalling that $f(\cdot)=\alpha\|\cdot\|$. Using the same arguments as in the proof of Lemma 3 we have that

$$
\dot{H} \leq-\tilde{q}_{\lambda}^{\top} K_{p} \Lambda \tilde{q}_{\lambda}-\left(\frac{\alpha}{2} \gamma-R_{\xi}\right)\left\|\sigma_{\lambda}\right\|<0,
$$

for all $\left(\sigma_{\lambda}, \tilde{q}_{\lambda}\right) \in \operatorname{bd}\left(W_{R, K_{p}}\right)$ which proves the positive invariance of $W_{R, K_{p}}$. Since $W_{R, K_{p}}$ is invariant we have that for any initial condition which lies in $W_{R, K_{p}}, \beta\left(\sigma_{\lambda}, \tilde{q}_{\lambda}\right)<R_{\xi}$ for all time $t \geq 0$ and consequently $\dot{H}<0$ for all $\sigma_{\lambda} \notin \alpha \lambda \mathbb{B}_{n}$ whenever $\alpha \gamma / 2>R_{\xi}$. It follows that $H$ will decrease at least until $\sigma_{\lambda}$ reaches the ball $\alpha \lambda \mathbb{B}_{n}$, this last fact also guarantees the convergence of $\tilde{q}_{\lambda}$ into a ball of appropriate radius $\rho$ (see proof of Lemma 3 ). In other words we have obtained semi-global practical asymptotic stability. 
It becomes clear that for any fixed $R>0$ and the conditions of Lemma 4 satisfied, application of Theorem 1 give us the existence of solutions of the differential inclusion (10) with the multivalued controller $u \in-\gamma \partial \Phi(\sigma)$.

\section{Robust stability of the closed-loop system}

In this section we prove how the trajectory tracking is achieved in a robust way against external bounded disturbances and parametric uncertainties. Additionally, we show that the variable $\sigma$ reaches zero in finite time. In order to get an upper bound of the reaching time, we shall take only the dynamic equation related to the $\sigma$ variable and we make $K_{p}=0$. i.e.,

$$
M(q) \dot{\sigma}+C(q, \dot{q}) \sigma+\xi(t, \sigma, \tilde{q}) \in-\gamma(\sigma, \tilde{q}) \partial \Phi(\sigma) .
$$

From the closed-loop equation (10) we see that the finite-time stabilization of the variable $\sigma$ implies the asymptotic stability of the error $\tilde{q}$ and its derivatives.

Theorem 2. Consider system (22). Let the assumptions of Theorem 1 hold. Let us set $\gamma(\sigma, \tilde{q})=(2 \beta(\sigma, \tilde{q})+\delta) / \alpha$, where $\delta>0$ is constant and $\beta$ as in Proposition 2. Then the sliding surface $\sigma=0$ is reached in finite time.

Proof. Consider the positive definite function of $\sigma, V(\sigma, t)=\frac{1}{2} \sigma^{\top} M(q(t)) \sigma$. Deriving $V$ along trajectories of (22) leads to

$$
\begin{aligned}
\dot{V} & =\frac{1}{2} \sigma^{\top} \dot{M}(q) \sigma+\sigma^{\top} M(q) \dot{\sigma} \\
& =\frac{1}{2} \sigma^{\top}(\dot{M}(q)-2 C(q, \dot{q})) \sigma-\langle\gamma(\sigma, \tilde{q}) \zeta+\xi(t, \sigma, \tilde{q}), \sigma\rangle, \\
& \leq-\gamma(\sigma, \tilde{q})\langle\zeta, \sigma\rangle+\|\xi(t, \sigma, \tilde{q})\|\|\sigma\|,
\end{aligned}
$$

where $\zeta \in \partial \Phi(\sigma)$ and we used Property 1. From subdifferential's definition together with Proposition 3 it follows that

$$
-\langle\zeta, \sigma\rangle \leq-\Phi(\sigma) \leq-\alpha\|\sigma\|,
$$

which yields the following expression

$$
\dot{V} \leq-[\alpha \gamma(\sigma, \tilde{q})-\beta(\sigma, \tilde{q})]\|\sigma\| .
$$

Hence, if $\alpha \gamma(\sigma, \tilde{q})=\beta(\sigma, \tilde{q})+\delta$ where $\delta=$ const. $>0$, we obtain $\dot{V} \leq-\delta\|\sigma\|=$ $-\delta \sqrt{\frac{2}{k_{2}}} V^{1 / 2}$. Separating variables and integrating the previous inequality over the time interval $[0, t]$, yields $V^{1 / 2}(t) \leq V^{1 / 2}(0)-\frac{\delta}{\sqrt{2 k_{2}}} t$, and consequently $V$ reaches zero in a finite time $t^{*}$ bounded by $t^{*} \leq \frac{\sqrt{2 k_{2}}}{\delta} V^{1 / 2}(0)$.

It is worth to mention that the multivalued control law (13) preserves the passivity of the closed-loop system as the following proposition shows.

Proposition 4. Let the assumptions of Theorem 1 hold. Then the closed-loop system (10)-(13) with new input $v$ (refer to Figure 1) without disturbances (i.e., $\xi \equiv 0$ ) is passive with input $v$ and output $\sigma$. 


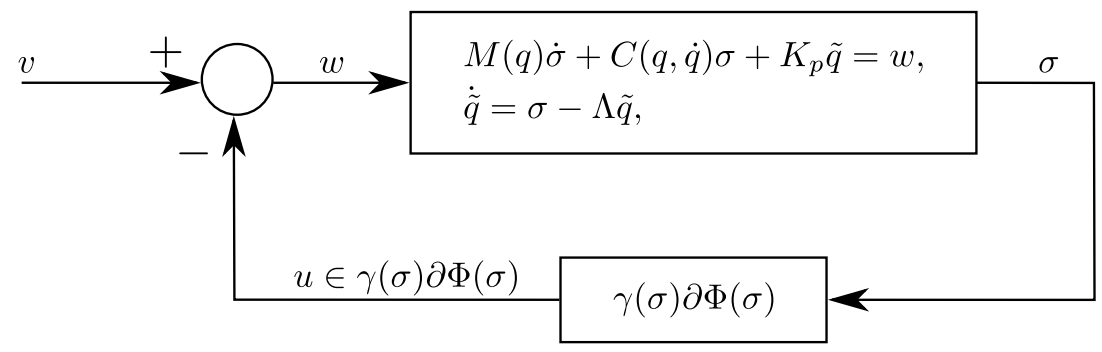

Figure 1: Closed-loop Lur'e system.

Proof. Consider the storage function $H(\sigma, \tilde{q})=\frac{1}{2} \sigma^{\top} M(q) \sigma+\frac{1}{2} \tilde{q}^{\top} K_{p} \tilde{q}$, then deriving along the trajectories of system (10) we have,

$$
\begin{aligned}
\dot{H}(\sigma, \tilde{q}) & =\frac{1}{2} \sigma^{\top} \dot{M}(q) \sigma+\sigma^{\top} M(q) \dot{\sigma}+\tilde{q}^{\top} K_{p} \dot{\tilde{q}} \\
& =\frac{1}{2} \sigma^{\top} \dot{M}(q) \sigma+\sigma^{\top}\left(v-\gamma(\sigma, \tilde{q}) \zeta-C(q, \dot{q}) \sigma-K_{p} \tilde{q}\right)+\tilde{q}^{\top} K_{p}(\sigma-\Lambda \tilde{q}) \\
& =\frac{1}{2} \sigma^{\top}(\dot{M}(q)-2 C(q, \dot{q})) \sigma-\tilde{q}^{\top} K_{p} \Lambda \tilde{q}-\gamma(\sigma, \tilde{q})\langle\zeta, \sigma\rangle+\sigma^{\top} v \\
& =-\tilde{q}^{\top} K_{p} \Lambda \tilde{q}-\alpha \gamma(\sigma, \tilde{q})\|\sigma\|+\sigma^{\top} v \\
& \leq \sigma^{\top} v
\end{aligned}
$$

where $\zeta$ is an element of $\partial \Phi(\sigma)$ and we use Property 1 together with the positiveness of $K_{p} \Lambda$ and Proposition 3.

This allows us to recast the set-valued closed-loop system, in the class of nonlinear nonsmooth Lur'e systems as depicted in Figure 1. Now that we have proved the global asymptotic stability of the origin in the presence of both parametric and external disturbances using a state-dependent gain $\gamma(\sigma, \tilde{q})$, we start looking for a constant gain $\gamma$, which as we can expect will depend of the initial conditions.

Theorem 3. Let the assumptions of Theorem 1 hold. Consider the system (10) with the multivalued control law $u \in-\gamma \partial \Phi(\sigma)$ and consider the compact set,

$$
W_{R, K_{p}}:=\left\{(\sigma, \tilde{q}) \in \mathbb{R}^{n} \times \mathbb{R}^{n} \mid H(\sigma, \tilde{q}) \leq R\right\},
$$

where

$$
H(\sigma, \tilde{q})=\frac{1}{2} \sigma^{\top} M(q) \sigma+\frac{1}{2} \tilde{q}^{\top} K_{p} \tilde{q} .
$$

Then for any initial condition $(\sigma(0), \tilde{q}(0))$ which lies in $W_{R, K_{p}}$ and any $R>0$ fixed, the origin of the closed-loop system is semi-globally asymptotically stable whenever

$$
\gamma \geq \frac{R_{\xi}}{\alpha}
$$

where $R_{\xi}$ is specified below in the proof.

Proof. From the fact that the set $W_{R, K_{p}}$ is positively invariant (the proof of this last fact is similar to the one of Lemma 4) and the fact that

$$
\begin{aligned}
\dot{H} & =-\tilde{q}^{\top} K_{p} \Lambda \tilde{q}-\gamma\langle\zeta, \sigma\rangle-\langle\xi(t, \sigma, \tilde{q}), \sigma\rangle \\
& \leq-\tilde{q}^{\top} K_{p} \Lambda \tilde{q}-\gamma \Phi(\sigma)+\beta(\sigma, \tilde{q})\|\sigma\| \\
& \leq-\tilde{q}^{\top} K_{p} \Lambda \tilde{q}-\left(\gamma \alpha-R_{\xi}\right)\|\sigma\|,
\end{aligned}
$$

is strictly negative whenever $\gamma>R_{\xi} / \alpha$, where $R_{\xi}=\max _{(\sigma, \tilde{q}) \in W_{R, K_{p}}} \beta(\sigma, \tilde{q})$ and $\zeta \in$ $\partial \Phi(\sigma)$. 


\section{Implicit discrete-time sliding-mode trajectory tracking control}

\subsection{Discrete-time controller design}

This section is devoted to the analysis of the discrete-time version of the above robust set-valued passivity-based control algorithms. An implicit discrete-time controller is used, as advocated in $[1,2,19]$ for linear time-invariant systems with known parameters, where equivalent-control-based sliding mode algorithms are analysed. The difficulty in extending the ideas in $[1,2,19]$ is that the plant is now nonlinear (thus preventing us from using an exact differentiation like the zero-order-hold method), the controller is also nonlinear, and most importantly we allow for parametric uncertainties. Let us start considering the following Euler discretized version of the plant (8):

$$
\left\{\begin{array}{l}
M\left(q_{k}\right) \frac{\dot{q}_{k+1}-\dot{q}_{k}}{h}+C\left(q_{k}, \dot{q}_{k}\right) \dot{q}_{k+1}+G\left(q_{k}\right)+F\left(t_{k}, q_{k}, \dot{q}_{k}\right)=\tau_{k}, \\
q_{k+1}=q_{k}+h \dot{q}_{k} .
\end{array}\right.
$$

It is worth to mention that Property 1 is fundamental for proving stability in the continuous-time formulation. Unfortunately it is not preserved exactly in the discretetime case, but a perturbed version is available instead. Henceforth for a function $F, F_{k}$ denotes $F\left(t_{k}\right)$.

In addition, assuming that the matrix $\hat{C}(q, \dot{q})$ is computed through the so-called Christoffel's symbols from $\hat{M}(q)$, then the pair $\hat{M}(q), \hat{C}(q, \dot{q})$ satisfies a property similar to Property 1.

Property 2. The matrices $\hat{M}(q)$ and $\hat{C}(q, \dot{q})$ satisfy

$$
\frac{d}{d t} \hat{M}(q(t))=\hat{C}(q(t), \dot{q}(t))+\hat{C}^{\top}(q(t), \dot{q}(t)) .
$$

It is worth to mention that Property 2 is not necessary in the continuous-time case since the explicit selection of the controller was not specified, contrary to the discrete-time setting where one of our main concerns is to obtain a numerical value for the control input at each time step (see (31)).

Lemma 5. For any $k \geq 0$ we have

$$
\begin{aligned}
& \hat{M}_{k+1}-\hat{M}_{k}=h \hat{C}_{k}+h \hat{C}_{k}^{\top}+\hat{\epsilon}_{k}, \\
& M_{k+1}-M_{k}=h C_{k}+h C_{k}^{\top}+\epsilon_{k},
\end{aligned}
$$

where $h$ is the so-called time step and satisfies $t_{k+1}-t_{k}=h$ and $\epsilon_{k}, \hat{\epsilon}_{k} \in \mathbb{R}^{n \times n}$ are $o(h)$ ('little-o') matrix functions, i.e.,

$$
\lim _{h \downarrow 0} \frac{\left\|\hat{\epsilon}_{k}\right\|_{m}}{h}=\lim _{h \downarrow 0} \frac{\left\|\epsilon_{k}\right\|_{m}}{h}=0 .
$$

Proof. Obtained from the expansion in Taylor series of Properties 1 and 2 [5].

Following the same methodology as in the continuous-time problem, we introduce the position error $\tilde{q}_{k}=q_{k}-q_{k}^{\mathrm{d}}$ as well as the sliding surface $\sigma_{k}=\dot{\tilde{q}}_{k}+\Lambda \tilde{q}_{k}$, where $\tilde{q}_{k+1}=$ $\tilde{q}_{k}+h \dot{\tilde{q}}_{k}, \Lambda \in \mathbb{R}^{n \times n}$ is a matrix whose spectrum is contained in the interior of the complex unitary circle, and $q_{k}^{\mathrm{d}}$ refers to the sample of the reference trajectory at time $t_{k}$. We propose the control law $\tau_{k}$ as

$$
\left\{\begin{array}{l}
\tau_{k}=\hat{M}_{k} \frac{\dot{q}_{k+1}^{r}-\dot{q}_{k}^{r}}{h}+\hat{C}_{k} \dot{q}_{k+1}^{r}+\hat{G}_{k}+u_{k} \\
q_{k+1}^{r}=q_{k}^{r}+h \dot{q}_{k}^{r}
\end{array}\right.
$$


where $\dot{q}_{k}^{r}=\dot{q}_{k}^{\mathrm{d}}-\Lambda \tilde{q}_{k}$ and $u_{k}$ refers to the multivalued part of the controller plus an additional dissipation term specified below. After some simple algebraic manipulations the first closed-loop system is obtained from (23) and (25) as

$$
\left\{\begin{array}{l}
M_{k} \sigma_{k+1}-M_{k} \sigma_{k}+h C_{k} \sigma_{k+1}+h \xi_{k}=h u_{k} \\
\tilde{q}_{k+1}=(I-h \Lambda) \tilde{q}_{k}+h \sigma_{k}
\end{array}\right.
$$

where $\sigma_{k+1}=\sigma_{k}+h \dot{\sigma}_{k}, \tilde{q}_{k+1}=\tilde{q}_{k}+h \dot{\tilde{q}}_{k}$, and the equivalent disturbance $\xi_{k}:=\xi\left(t_{k}, \sigma_{k}, \tilde{q}_{k}\right)$ is given by

$$
\begin{aligned}
\xi_{k}=F_{k}+\left(M_{k}-\hat{M}_{k}\right) & \left(\ddot{q}_{k}^{d}-\Lambda\left(\sigma_{k}-\Lambda \tilde{q}_{k}\right)\right) \\
& +\left(C_{k}-\hat{C}_{k}\right)\left(\dot{q}_{k+1}^{\mathrm{d}}-\Lambda\left[(I-h \Lambda) \tilde{q}_{k}+h \sigma_{k}\right]\right)+G_{k}-\hat{G}_{k} .
\end{aligned}
$$

It is easy to prove that the discrete-time version of the disturbance $\xi_{k}$ satisfies an analogue version of Proposition 2 as:

Proposition 5. The function $\xi\left(t_{k}, \sigma_{k}, \tilde{q}_{k}\right)$ satisfies

$$
\left\|\xi\left(t_{k}, \sigma_{k}, \tilde{q}_{k}\right)\right\| \leq \beta\left(\sigma_{k}, \tilde{q}_{k}\right)
$$

where $\beta\left(\sigma_{k}, \tilde{q}_{k}\right)=c_{1}+c_{2}\left\|\sigma_{k}\right\|+c_{3}\left\|\tilde{q}_{k}\right\|+c_{4}\left\|\tilde{q}_{k}\right\|\left\|\sigma_{k}\right\|+c_{5}\left\|\tilde{q}_{k}\right\|^{2}$, for bounded positive constants $c_{i}, i=1, \ldots, 5$.

Proof. The proof is omitted because it is obtained following the same steps as in the proof of Proposition 2.

It is worth to mention that the control law $\tau_{k}$ depends only of the data available at time $t_{k}$ as well as the position and velocity errors, whenever $u_{k}$ is also well-posed and non anticipative. Simple computations reveal that (25) is equivalent to

$$
\tau_{k}=\hat{M}_{k}\left(\ddot{q}_{k}^{\mathrm{d}}-\Lambda\left(\sigma_{k}-\Lambda \tilde{q}_{k}\right)\right)+\hat{C}_{k}\left(\dot{q}_{k+1}^{\mathrm{d}}-\Lambda\left[(I-h \Lambda) \tilde{q}_{k}+h \sigma_{k}\right]\right)+\hat{G}_{k}+u_{k} .
$$

Additionally, from (26b) we are led to make the following assumption,

Assumption 8. The step length $h>0$ is small enough such that the spectrum of $I-h \Lambda$ is contained in the interior of the complex unitary circle.

At this point we specify the missing term $u_{k}$ in a similar way as its counterpart in continuous-time (13) as

$$
-u_{k} \in K_{\sigma} \hat{\sigma}_{k+1}+\gamma \partial \Phi\left(\hat{\sigma}_{k+1}\right),
$$

where $K_{\sigma}=K_{\sigma}^{\top}>0, \gamma>0$ is considered constant, and $\hat{\sigma}_{k+1}$ is related to the nominal version of (26a) as

$$
\hat{M}_{k} \hat{\sigma}_{k+1}-\hat{M}_{k} \sigma_{k}+h \hat{C}_{k} \hat{\sigma}_{k+1}+h K_{\sigma} \hat{\sigma}_{k+1} \in-h \gamma \partial \Phi\left(\hat{\sigma}_{k+1}\right) .
$$

Since the equivalent disturbance $\xi_{k}$ is unknown, we will calculate the controller from the nominal unperturbed plant (30) with state $\hat{\sigma}_{k}$, and using (26), as follows:

$$
\left\{\begin{array}{l}
M_{k} \sigma_{k+1}-M_{k} \sigma_{k}+h C_{k} \sigma_{k+1}+h K_{\sigma} \hat{\sigma}_{k+1}+h \xi_{k}=-h \gamma \zeta_{k+1}, \\
\zeta_{k+1} \in \partial \Phi\left(\hat{\sigma}_{k+1}\right) \\
\hat{M}_{k} \hat{\sigma}_{k+1}-\hat{M}_{k} \sigma_{k}+h \hat{C}_{k} \hat{\sigma}_{k+1}+h K_{\sigma} \hat{\sigma}_{k+1}=-h \gamma \zeta_{k+1} \\
\tilde{q}_{k+1}=(I-h \Lambda) \tilde{q}_{k}+h \sigma_{k}
\end{array}\right.
$$


Notice that the discrete-time closed-loop system (31) is slightly different from the direct discretization of the continuous-time closed-loop system (10), by adding a new term $K_{\sigma} \hat{\sigma}_{k+1}$ and making $K_{p}=0$. The additional term $K_{\sigma} \hat{\sigma}_{k+1}$ will allow us to assure the stability of the closed-loop system by adding dissipation, as is shown in the proofs of Theorems 4 and 5. From now on we will concentrate our attention on equations (31a)(31c) for which if some stability result is preserved, then the boundedness of the solution of the difference equation (31d) is guaranteed as will be shown later. Moreover, from Assumption 8 we have that $\tilde{q}_{k} \rightarrow 0$ as $\sigma_{k} \rightarrow 0$ and $k \rightarrow+\infty$. System (31a)-(31d) may be viewed as follows: (31a) and (31d) are the Euler discretization of the plant with a pre-feedback, (31c) is a nominal unperturbed system, and (31b) is the discretized setvalued controller to be calculated from (31c). From (31) it becomes clear that when all uncertainties and disturbances vanish, then $\hat{\sigma}_{k}=\sigma_{k}$, whenever $\hat{\sigma}_{0}=\sigma_{0}$.

Remark 5. The set-valued controller is approximated in (29) with an implicit approach. An explicit discretization would yield $\partial \Phi\left(\sigma_{k}\right)$ instead, since $\sigma_{k}$ is known at $t=t_{k}$. Explicit discretizations however yield the so-called numerical chattering effect on both input and output [14, 17, 18, 40, 41].

First, we prove the well-posedness of the general scheme (31), i.e., we prove that we can compute a selection of the multivalued controller (31b) in a unique fashion, using only the information available at the time step $k$. To this end we have from (31c) and (31b),

$$
\left(\hat{M}_{k}+h \hat{C}_{k}+h K_{\sigma}\right) \hat{\sigma}_{k+1}-\hat{M}_{k} \sigma_{k} \in-h \gamma \partial \Phi\left(\hat{\sigma}_{k+1}\right) .
$$

Equivalently,

$$
\left\langle\hat{\mathcal{A}}_{k} \hat{\sigma}_{k+1}-\hat{M}_{k} \sigma_{k}, \eta-\hat{\sigma}_{k+1}\right\rangle+h \gamma \Phi(\eta)-h \gamma \Phi\left(\hat{\sigma}_{k+1}\right) \geq 0, \text { for all } \eta \in \mathbb{R}^{n},
$$

where $\hat{\mathcal{A}}_{k}:=\left(\hat{M}_{k}+h \hat{C}_{k}+h K_{\sigma}\right)$. It becomes clear in the light of Lemma 1 that $\hat{\sigma}_{k+1}$ is uniquely determined if the operator $\hat{\mathcal{A}}_{k}$ is strongly monotone. Additionally, it is also worth to mention that such $\hat{\sigma}_{k+1}$ depends on $\hat{\mathcal{A}}_{k}, \hat{M}_{k}, \sigma_{k}, h, \gamma$ and $\Phi$ (all them available at time step $k$ ). For the strong monotonicity of $\hat{\mathcal{A}}_{k}$, we have that for any $w \in \mathbb{R}^{n}$ the following holds,

$$
\begin{aligned}
\left\langle\hat{\mathcal{A}}_{k} w, w\right\rangle & =w^{\top}\left(\hat{M}_{k}+h K_{\sigma}\right) w+\frac{1}{2} w^{\top}\left(\hat{M}_{k+1}-\hat{M}_{k}-\hat{\epsilon}_{k}\right) w \\
& \geq\left(\hat{k}_{1}+h \kappa_{1}-\frac{\left\|\hat{\epsilon}_{k}\right\|_{m}}{2}\right)\|w\|^{2}
\end{aligned}
$$

where $\kappa_{1}$ is the minimum eigenvalue of the matrix $K_{\sigma}$ and we made use of Assumption 6 and Lemma 5. Hence, for any $h$ sufficiently small such that

$$
\frac{\hat{k}_{1}}{2}+h \kappa_{1}-\frac{\left\|\hat{\epsilon}_{k}\right\|_{m}}{2} \geq 0
$$

it follows that $\hat{\mathcal{A}}_{k}$ is strongly monotone. Therefore, by applying Lemma 1 we obtain the uniqueness of $\hat{\sigma}_{k+1}$. Moreover, the solution $\hat{\sigma}_{k+1}$ is Lipschitz continuous with respect to $\sigma_{k}$. It is worth to mention that the condition on the strong monotonicity of $\hat{\mathcal{A}}_{k}$ of Lemma 1 can be relaxed using the approach developed in [3], especially the results in Section 2.7 (see also [27]). The following Lemma allows us to obtain an implicit expression for the solution of (33) which can be solved numerically in an easy way.

Lemma 6. Consider the following variational inequality of second kind

$$
\langle P x-r, \eta-x\rangle+\phi(\eta)-\phi(x) \geq 0, \text { for all } \eta \in \mathbb{R}^{n},
$$


with $P \in \mathbb{R}^{n \times n}$ a strongly monotone operator (but no necessarily symmetric). Then the unique solution of (36) satisfies

$$
x=\operatorname{Prox}_{\mu \phi}((I-\mu P) x+\mu r)=\left(I d-\mu \operatorname{Prox}_{\phi^{*} / \mu} \circ \mu^{-1} I d\right)((I-\mu P) x+\mu r)
$$

for some $\mu>0$. Moreover, the map $x \mapsto \operatorname{Prox}_{\mu \phi}((I-\mu P) x+\mu r)$ is a contraction for $\mu$ small enough.

Proof. Let $x$ be the solution of (36). Then for any $\mu>0$ we have

$$
\mu r-\mu P x \in \partial(\mu \phi)(x) \Leftrightarrow(I-\mu P) x+\mu r-x \in \partial(\mu \phi)(x) .
$$

Hence $x=\operatorname{Prox}_{\mu \phi}((I-\mu P) x+\mu r)$. The second equality in (37) is a direct consequence of the Moreau's decomposition Theorem (Proposition 1). Recalling that Prox ${ }_{\mu \phi}$ is a nonexpansive operator, we have that

$$
\begin{aligned}
\left\|\operatorname{Prox}_{\mu \phi}\left((I-\mu P) x_{1}+\mu r\right)-\operatorname{Prox}_{\mu \phi}\left((I-\mu P) x_{2}+\mu r\right)\right\| & \leq\left\|(I-\mu P)\left(x_{1}-x_{2}\right)\right\| \\
& \leq\|I-\mu P\|_{m}\left\|x_{1}-x_{2}\right\| .
\end{aligned}
$$

Now, because we are using the Euclidean norm we have that the induced norm of a matrix $A$ satisfies $\|A\|_{m}=\sqrt{\lambda_{\max }\left(A^{\top} A\right)}$ (see e.g., [25, p. 365 Exercise 5]). Thus, it follows that if $(I-\mu P)^{\top}(I-\mu P)<I$, where the order relation $<$ means that $I-(I-$ $\mu P)^{\top}(I-\mu P)$ is positive definite, then the map defined by $x \mapsto \operatorname{Prox}_{\mu \phi}((I-\mu P) x+\mu r)$ is a contraction. Therefore,

$$
0<I-(I-\mu P)^{\top}(I-\mu P) \Leftrightarrow 0<P+P^{\top}-\mu P^{\top} P,
$$

and from the strong monotonicity property of $P$ it follows that selecting $\mu$ small enough we arrive at the desired conclusion.

Remark 6. There are several ways to numerically solve problems like (36), like the semismooth Newton method [13, §7.5] as advocated in [5, Section 6]. For control applications this method may be time consuming, since it involves the computation of inverse matrices and proximal maps of composite functions. In contrast, the simple method of successive approximations [23, §14] can be applied in order to find quickly the fixed point in (37). Details about the implementation are given in Section $\%$

From Lemma 6 the selection of the control value can be obtained from (31b) (31c) as

$$
\begin{aligned}
\zeta_{k+1} & =-\frac{1}{h \gamma}\left(\hat{\mathcal{A}}_{k} \hat{\sigma}_{k+1}-\hat{M}_{k} \sigma_{k}\right) \\
\hat{\sigma}_{k+1} & =\operatorname{Prox}_{\mu h \gamma \Phi}\left(\left(I-\mu \hat{\mathcal{A}}_{k}\right) \hat{\sigma}_{k+1}+\mu \hat{M}_{k} \sigma_{k}\right),
\end{aligned}
$$

for any $\mu>0$ such that

$$
0<\hat{\mathcal{A}}_{k}+\hat{\mathcal{A}}_{k}^{\top}-\mu \hat{\mathcal{A}}_{k}^{\top} \hat{\mathcal{A}}_{k} .
$$

The solution of the implicit equation (38b) with unknown $\hat{\sigma}_{k+1}$ being a function of $\sigma_{k}$ and $h$, it is clear from (38a) that the controller is non-anticipative. Let us now show conditions that guarantee (35), consequently the possible application of Lemma 6 to (33).

Lemma 7. There exists $\delta^{*}>0$ (which is a function of $\tilde{q}_{0}$ and $\sigma_{0}$ ) such that for any $h \in\left(0, \delta^{*}\right]$ the following inequalities hold

$$
\begin{gathered}
\left\|\hat{\epsilon}_{k}\right\|_{m} \leq \min \left\{\hat{k}_{1}, 2 h \kappa_{1}\right\}, \\
\left\|\epsilon_{k}\right\|_{m} \leq \min \left\{k_{1}, 2 h \kappa_{1}\right\},
\end{gathered}
$$

where $\kappa_{1}$ is the minimum eigenvalue of $K_{\sigma}$ and $\hat{\epsilon}_{k}, \epsilon_{k}$ satisfy (24). 
Proof. By Lemma 5 it follows that (40) is always solvable. Indeed, since $\hat{\epsilon}_{k}\left(\epsilon_{k}\right)$ is $o(h)$ we have that for any $\hat{\varepsilon}>0(\varepsilon>0)$ there exists $\hat{\delta}>0(\delta>0)$ such that $\left\|\hat{\epsilon}_{k}\right\|_{m}<\hat{\varepsilon} h$ $(\|\varepsilon\|<\varepsilon h)$ for all $h<\hat{\delta}(h<\delta)$. Therefore, choosing $\hat{\varepsilon}$ and $\varepsilon$ small enough, we have that for any $h \in(0, \min \{\hat{\delta}, \delta\}]$ both inequalities in (40) are fulfilled.

There is one detail to consider in the previous reasoning related with the uniformity of $h$, i.e., $\delta^{*}$ and $\hat{\delta}$ can be selected independently of the step $k$. This fact becomes more clear after proving that all solutions of (31) are bounded. The strict reader can think as $h=h_{k}$, see Remark 9. Moreover, from Lemma 7 it is easy to see that (40a) implies $(35)$.

\subsection{Stability of the discrete-time closed-loop system}

The second important question to solve, after the input calculation, is about the stability of the full discrete-time system (31). To this end we present two cases. The first one attacks the stability issue without parametric uncertainty, whereas in the second case the full perturbation case (i.e., external disturbance and parametric uncertainty) is considered. The following bounds will be useful.

Proposition 6. Let Assumption 6 hold, and assume that the time step $h>0$ is such that (40a) is satisfied. Then, for all $k \in \mathbb{N}$, the following bounds hold:

$$
\begin{aligned}
\left\|\hat{\mathcal{A}}_{k}^{-1}\right\|_{m} & \leq \frac{1}{\hat{k}_{1}} \\
\left\|\hat{\mathcal{B}}_{k}^{-1}\right\|_{m} & \leq \frac{2}{\hat{k}_{1}}
\end{aligned}
$$

where $\hat{\mathcal{A}}_{k}:=\hat{M}_{k}+h \hat{C}_{k}+h K_{\sigma}$ and $\hat{\mathcal{B}}_{k}:=\hat{M}_{k}+h \hat{C}_{k}$.

Proof. From (34) and (40a) we have that for any $w \in \mathbb{R}^{n} \backslash\{0\}$

$$
\begin{aligned}
\left\|\left(\hat{M}_{k}+h \hat{C}_{k}+h K_{\sigma}\right) w\right\|\|w\| & \geq\left\langle\left(\hat{M}_{k}+h \hat{C}_{k}+h K_{\sigma}\right) w, w\right\rangle \\
& \geq\left(\hat{k}_{1}+h \kappa_{1}-\frac{\left\|\hat{\epsilon}_{k}\right\|_{m}}{2}\right)\|w\|^{2} \\
& \geq \hat{k}_{1}\|w\|^{2}
\end{aligned}
$$

and then

$$
\|w\| \leq \frac{1}{\hat{k}_{1}}\left\|\left(\hat{M}_{k}+h \hat{C}_{k}+h K_{\sigma}\right) w\right\| .
$$

Setting $w=\left(\hat{M}_{k}+h \hat{C}_{k}+h K_{\sigma}\right)^{-1} x$, where $x \in \mathbb{R}^{n} \backslash\{0\}$, yields the desired result. The proof of the second inequality follows the same steps and the fact that $\hat{k}_{1}-\|\hat{\epsilon}\|_{m} \geq 0$ (see (40a)). Indeed,

$$
\begin{aligned}
\left\|\left(\hat{M}_{k}+h \hat{C}_{k}\right) w\right\|\|w\| & \geq\left\langle\left(\hat{M}_{k}+h \hat{C}_{k}\right) w, w\right\rangle \geq\left(\hat{k}_{1}-\frac{\left\|\hat{\epsilon}_{k}\right\|_{m}}{2}\right)\|w\|^{2} \\
& \geq \frac{\hat{k}_{1}}{2}\|w\|^{2},
\end{aligned}
$$

Setting $w=\left(\hat{M}_{k}+h \hat{C}_{k}\right)^{-1} x$, finishes the proof.

Remark 7. Notice that in an analogue way we also obtain bounds for matrices $\mathcal{A}_{k}^{-1}$ and $\mathcal{B}_{k}^{-1}$ calculated with $M_{k}$ and $C_{k}$, as $\left\|\mathcal{A}_{k}^{-1}\right\|_{m} \leq \frac{1}{k_{1}}$ and $\left\|\mathcal{B}_{k}^{-1}\right\|_{m} \leq \frac{2}{k_{1}}$ by making use of (40b) in Proposition 6 instead. 
Before presenting the main results about stability of the closed-loop system (31) we show that even in the presence of an external perturbation $\xi_{k}$, the variable $\hat{\sigma}_{k}$ which is the state of the nominal unperturbed system (31b)-(31c), can be maintained at zero.

Lemma 8. Let $h>0$ be small enough such that (40a) hold. If

$$
\left\|\frac{\hat{M}_{k} \sigma_{k}}{h}\right\| \leq \gamma \alpha,
$$

then $\hat{\sigma}_{k+1}=0$. Moreover, in the case where there is no parametric uncertainty (i.e., $M_{k}=\hat{M}_{k}, C_{k}=\hat{C}_{k}$ and $\xi_{k}$ is uniformly bounded by some constant $\left.0<\bar{F}<+\infty\right)$, if $\hat{\sigma}_{k_{0}+1}=0$ for some $k=k_{0}$ then $\hat{\sigma}_{k_{0}+n}=0$ for all $n \geq 1$ whenever,

$$
2 \frac{\hat{k}_{2}}{\hat{k}_{1}} \bar{F} \leq \gamma \alpha
$$

Proof. By the uniqueness of solution of the variational inequality (33), we have that $\hat{\sigma}_{k+1}=0$ if and only if $\frac{\hat{M}_{k} \sigma_{k}}{h \gamma}$ belongs to the set of minimizers of the conjugate function $\Phi^{\star}$. Indeed from (32),

$$
\begin{aligned}
\hat{\sigma}_{k+1}=0 & \Leftrightarrow \frac{\hat{M}_{k} \sigma_{k}}{h \gamma} \in \partial \Phi(0) \Leftrightarrow 0 \in \partial \Phi^{\star}\left(\frac{\hat{M}_{k} \sigma_{k}}{h \gamma}\right) \\
& \Leftrightarrow \frac{\hat{M}_{k} \sigma_{k}}{h \gamma} \in \operatorname{Arg} \min \Phi^{\star} .
\end{aligned}
$$

Now, under Assumption 7 we have that $\Phi(\cdot) \geq \alpha\|\cdot\|$ which in fact implies $\Psi_{\alpha \mathbb{B}_{n}}(\cdot) \geq$ $\Phi^{\star}(\cdot)$. Recall that the conjugate function of $\alpha\|\cdot\|$ is the indicator function of the set $\alpha \mathbb{B}_{n}$ and $f \geq g$ implies $g^{\star} \geq f^{\star}$. Hence, we have that for any $w \in \alpha \mathbb{B}_{n}, \Phi^{\star}(w) \leq 0$. On the other hand, from the definition of the conjugate function, the fact that $\Phi \in \Gamma_{0}\left(\mathbb{R}^{n}\right)$ and using the Fenchel-Moreau Theorem [9, Theorem I.10], it is easy to deduce that $0=\Phi(0)=\Phi^{\star \star}(0)=-\inf \Phi^{\star}$, and we have $0 \leq \Phi^{\star}(w)$ for all $w \in \mathbb{R}^{n}$. Therefore we have proved that for any $w \in \alpha \mathbb{B}_{n}$, one has $\Phi^{\star}(w)=0$, while $\Phi^{\star}(\cdot) \geq 0$ everywhere. So $\alpha \mathbb{B}_{n} \subseteq \operatorname{Arg} \min \Phi^{\star}$. This finishes the first part of the proof.

For the second part, let $k_{0}$ be such that $\hat{\sigma}_{k_{0}+1}=0$. Then from (31c) we obtain $-\hat{M}_{k} \sigma_{k_{0}}=$ $-h \gamma \zeta_{k_{0}+1}$ for some $\zeta_{k_{0}+1} \in \partial \Phi(0)$. Substitution of $\sigma_{k_{0}}$ in (31a) gives:

$$
\sigma_{k_{0}+1}=-h \hat{\mathcal{B}}_{k_{0}}^{-1} \xi_{k_{0}} .
$$

Hence, the use of (42) together with (43) yields

$$
\left\|\frac{\hat{M}_{k_{0}+1} \sigma_{k_{0}+1}}{h}\right\|=\left\|\hat{M}_{k_{0}+1} \hat{\mathcal{B}}_{k_{0}}^{-1} \xi_{k_{0}}\right\| \leq \frac{2 \hat{k}_{2}}{\hat{k}_{1}} \bar{F} \leq \gamma \alpha .
$$

Therefore from the above we get $\hat{\sigma}_{k_{0}+2}=0$. Following with the proof by induction we conclude that $\hat{\sigma}_{k_{0}+n}=0$ for all $n \geq 1$.

In continous-time the selection of the set-valued controller exactly compensates for the perturbation on the sliding surface $\sigma(t)=0$, see (22). An interesting issue is about the value of the discrete-time controller once the "nominal state sliding surface" $\hat{\sigma}_{k}=0$ has been attained.

Corollary 1. Under assumptions of Lemma 8, the equivalent control which maintains $\hat{\sigma}_{k+n}=0$ for all $n \geq 1$ is given by

$$
\zeta_{k+2}^{e q}=\frac{1}{h \gamma} \hat{M}_{k+1} \mathcal{B}_{k}^{-1}\left(\left(M_{k}-\hat{M}_{k}\right) \sigma_{k}-h \xi_{k}\right)
$$

where $\mathcal{B}_{k}=M_{k}+h C_{k}$. 
Proof. Under the assumption that $\hat{\sigma}_{k+n}=0$ for all $n \geq 1$, from (31c) we obtain $\hat{M}_{k} \sigma_{k}=$ $-h \gamma \zeta_{k+1}^{e q}$. Substitution of $\zeta_{k+1}^{e q}$ in (31a) yields

$$
\left(M_{k}+h C_{k}\right) \sigma_{k+1}-\left(M_{k}-\hat{M}_{k}\right) \sigma_{k}=-h \xi_{k} .
$$

It follows that $\sigma_{k+1}=\mathcal{B}_{k}^{-1}\left(\left(M_{k}-\hat{M}_{k}\right) \sigma_{k}-h \xi_{k}\right)$. Thus, one iteration through (31c) results in $\hat{\mathcal{A}}_{k} \hat{\sigma}_{k+2}-\hat{M}_{k+1} \sigma_{k+1}=-h \gamma \zeta_{k+2}^{e q}$, and the result follows.

The previous Corollary shows that the scheme in (38) for computing the controller from the nominal system (31b)-(31c) allows to compensate for the disturbance with a delay of two time steps. Obviously the equivalent control in (45) is not implementable since the disturbance is unknown. It is noteworthy that the magnitude of the equivalent control in (45) does not depend on $h$, see (44).

Theorem 4 (No parametric uncertainty). Let Assumptions 1-8 hold. Consider the discrete-time dynamical system (31) without parametric uncertainty (i.e., $M_{k}=\hat{M}_{k}$, $C_{k}=\hat{C}_{k}$ and $\xi_{k}$ uniformly bounded by $\left.\bar{F}\right)$. Then for any initial condition $\sigma_{0} \in \mathbb{R}^{n}$ the solution $\sigma_{k}$ of (31a) converges to a ball of radius $\frac{2 \bar{F}}{\hat{k}_{1}} h$ in a finite number of steps, i.e., the system is globally practically stable, whenever,

$$
\gamma \alpha \geq \max \left\{\frac{2 \hat{k}_{2}}{\hat{k}_{1}} \bar{F}\left(1+\frac{\bar{F}}{\hat{k}_{1} \hat{r}}\right), 2 \hat{k}_{2} \sqrt{\frac{\hat{k}_{2}}{\hat{k}_{1}}}\left(\hat{r}+\frac{2 \bar{F}}{\hat{k}_{1}}\right)\right\}
$$

for some $0<\hat{r}$ small enough and fixed. Moreover, $\hat{\sigma}_{k}$ reaches the origin in a finite number of steps $k^{*}$, and $\hat{\sigma}_{k}=0$ for all $k \geq k^{*}+1$.

Proof. Consider the functions $V_{1, k}:=\hat{\sigma}_{k}^{\top} \hat{M}_{k} \hat{\sigma}_{k}$ and $V_{2, k}:=\sigma_{k}^{\top} \hat{M}_{k} \sigma_{k}$ and their respective differences $\Delta V_{i}:=V_{i, k+1}-V_{i, k}$, for $i=1,2$. It follows from (24) and (31c) that the next chain of relations holds,

$$
\begin{aligned}
\Delta V_{1}= & \hat{\sigma}_{k+1}^{\top} \hat{M}_{k+1} \hat{\sigma}_{k+1}-\hat{\sigma}_{k}^{\top} \hat{M}_{k} \hat{\sigma}_{k} \\
= & \hat{\sigma}_{k+1}^{\top}\left(\hat{M}_{k+1}-\hat{M}_{k}\right) \hat{\sigma}_{k+1}-\hat{\sigma}_{k}^{\top} \hat{M}_{k} \hat{\sigma}_{k}+\hat{\sigma}_{k+1}^{\top} \hat{M}_{k} \hat{\sigma}_{k+1} \\
= & \hat{\sigma}_{k+1}^{\top}\left(\hat{M}_{k+1}-\hat{M}_{k}\right) \hat{\sigma}_{k+1}+\hat{\sigma}_{k+1}^{\top} \hat{M}_{k}\left(\hat{\sigma}_{k+1}-\sigma_{k}\right)+\hat{\sigma}_{k+1}^{\top} \hat{M}_{k} \sigma_{k}-\hat{\sigma}_{k} \hat{M}_{k} \hat{\sigma}_{k} \\
= & \hat{\sigma}_{k+1}^{\top}\left(\hat{M}_{k+1}-\hat{M}_{k}\right) \hat{\sigma}_{k+1}+2 \hat{\sigma}_{k+1}^{\top} \hat{M}_{k}\left(\hat{\sigma}_{k+1}-\sigma_{k}\right)-\hat{\sigma}_{k+1}^{\top} \hat{M}_{k} \hat{\sigma}_{k+1} \\
& +2 \hat{\sigma}_{k+1}^{\top} \hat{M}_{k} \sigma_{k}-\hat{\sigma}_{k}^{\top} \hat{M}_{k} \hat{\sigma}_{k} \\
\leq & \hat{\sigma}_{k+1}^{\top}\left(2 h \hat{C}_{k}+\hat{\epsilon}_{k}\right) \hat{\sigma}_{k+1}+2 \hat{\sigma}_{k+1}^{\top}\left(-h \hat{C}_{k} \hat{\sigma}_{k+1}-h K_{\sigma} \hat{\sigma}_{k+1}-h \gamma \zeta_{k+1}\right) \\
& +\sigma_{k}^{\top} \hat{M}_{k} \sigma_{k}-\hat{\sigma}_{k}^{\top} \hat{M}_{k} \hat{\sigma}_{k},
\end{aligned}
$$

where we apply the inequality $2 x^{\top} Q y \leq x^{\top} Q x+y^{\top} Q y$ and $Q=Q^{\top}>0$ to the term $2 \hat{\sigma}_{k+1}^{\top} \hat{M}_{k} \sigma_{k}$. Now, adding and substracting the term $\sigma_{k+1}^{\top} \hat{M}_{k+1} \sigma_{k+1}+\hat{\sigma}_{k+1}^{\top} \hat{M}_{k+1} \hat{\sigma}_{k+1}$ in the right-hand side of (47) results in

$$
\begin{aligned}
\Delta V_{1} \leq \hat{\sigma}_{k+1}^{\top} \hat{\epsilon}_{k} \hat{\sigma}_{k+1}-2 h \hat{\sigma}_{k+1}^{\top}\left(K_{\sigma} \hat{\sigma}_{k+1}+\gamma \zeta_{k+1}\right) & -\Delta V_{2}+\Delta V_{1} \\
& +\sigma_{k+1}^{\top} \hat{M}_{k+1} \sigma_{k+1}-\hat{\sigma}_{k+1}^{\top} \hat{M}_{k+1} \hat{\sigma}_{k+1},
\end{aligned}
$$

and it follows that

$$
\begin{aligned}
\Delta V_{2} \leq \hat{\sigma}_{k+1}^{\top} \hat{\epsilon}_{k} \hat{\sigma}_{k+1}-2 h \hat{\sigma}_{k+1}^{\top}\left(K_{\sigma} \hat{\sigma}_{k+1}+\gamma \zeta_{k+1}\right) & \\
& +\sigma_{k+1}^{\top} \hat{M}_{k+1} \sigma_{k+1}-\hat{\sigma}_{k+1}^{\top} \hat{M}_{k+1} \hat{\sigma}_{k+1}
\end{aligned}
$$


On the other hand, the substitution of (31c) into (31a) yields (recall that here $M_{k}=\hat{M}_{k}$, $C_{k}=\hat{C}_{k}$, and $\hat{\mathcal{B}}_{k}=\hat{M}_{k}+h \hat{C}_{k}$ )

$$
\sigma_{k+1}=\hat{\sigma}_{k+1}-h \hat{\mathcal{B}}_{k}^{-1} \xi_{k}
$$

Hence, simple computations give:

$$
\begin{aligned}
\sigma_{k+1}^{\top} \hat{M}_{k+1} \sigma_{k+1} & =\left(\hat{\sigma}_{k+1}-h \hat{\mathcal{B}}_{k}^{-1} \xi_{k}\right)^{\top} \hat{M}_{k+1}\left(\hat{\sigma}_{k+1}-h \hat{\mathcal{B}}_{k}^{-1} \xi_{k}\right) \\
& =\hat{\sigma}_{k+1} \hat{M}_{k+1} \hat{\sigma}_{k+1}-2 h \hat{\sigma}_{k+1}^{\top} \hat{M}_{k+1} \mathcal{B}_{k}^{-1} \xi_{k}+h^{2} \xi^{\top} \mathcal{B}_{k}^{-\top} \hat{M}_{k+1} \mathcal{B}_{k}^{-1} \xi_{k} .
\end{aligned}
$$

Thus, after substitution of (50) into (48) it follows that (recall that $\kappa_{1}=\lambda_{\min }\left(K_{\sigma}\right)$ )

$$
\begin{aligned}
\Delta V_{2} \leq & \left\|\hat{\epsilon}_{k}\right\|_{m}\left\|\hat{\sigma}_{k+1}\right\|^{2}-2 h \hat{\sigma}_{k+1}^{\top}\left(K_{\sigma} \hat{\sigma}_{k+1}+\gamma \zeta_{k+1}\right) \\
& -2 h \hat{\sigma}_{k+1}^{\top} \hat{M}_{k+1} \mathcal{B}_{k}^{-1} \xi_{k}+h^{2} \xi^{\top} \mathcal{B}_{k}^{-\top} \hat{M}_{k+1} \mathcal{B}_{k}^{-1} \xi_{k} \\
\leq & -\left(2 h \kappa_{1}-\|\hat{\epsilon}\|_{m}\right)\left\|\hat{\sigma}_{k+1}\right\|^{2}-2 h \gamma \hat{\sigma}_{k+1}^{\top} \zeta_{k+1}+4 h \frac{\hat{k}_{2}}{\hat{k}_{1}}\left\|\xi_{k}\right\|\left\|\hat{\sigma}_{k+1}\right\|+4 h^{2} \frac{\hat{k}_{2}}{\hat{k}_{1}^{2}}\left\|\xi_{k}\right\|^{2} \\
\leq & -\left(2 h \kappa_{1}-\|\hat{\epsilon}\|_{m}\right)\left\|\hat{\sigma}_{k+1}\right\|^{2}-2 h\left(\gamma \alpha-\frac{2 \hat{k}_{2}}{\hat{k}_{1}} \bar{F}\right)\left\|\hat{\sigma}_{k+1}\right\|+4 h^{2} \frac{\hat{k}_{2}}{\hat{k}_{1}^{2}} \bar{F}^{2}
\end{aligned}
$$

where we used the fact that $\zeta_{k+1} \in \partial \Phi\left(\hat{\sigma}_{k+1}\right)$ together with Proposition 3 in the last inequality. Now assume that $\left\|\sigma_{k+1}\right\|>\left(\hat{r}+\frac{2 \bar{F}}{\hat{k}_{1}}\right) h$ for some $0<\hat{r}<+\infty$. From (49) and (42) it follows that $\left\|\sigma_{k+1}\right\|>\left(\hat{r}+\frac{2 \bar{F}}{\hat{k}_{1}}\right) h$ implies $\left\|\hat{\sigma}_{k+1}\right\|>\hat{r} h$. Hence,

$$
\begin{aligned}
\Delta V_{2} \leq & -\left(2 h \kappa_{1}-\|\hat{\epsilon}\|_{m}\right)\left\|\hat{\sigma}_{k+1}\right\|^{2}-2 h\left(\gamma \alpha-\frac{2 \hat{k}_{2}}{\hat{k}_{1}} \bar{F}\right)\left\|\hat{\sigma}_{k+1}\right\|+4 h^{2} \frac{\hat{k}_{2}}{\hat{k}_{1}^{2}\left\|\hat{\sigma}_{k+1}\right\|} \bar{F}^{2}\left\|\hat{\sigma}_{k+1}\right\| \\
\leq & -\left(2 h \kappa_{1}-\|\hat{\epsilon}\|_{m}\right)\left\|\hat{\sigma}_{k+1}\right\|^{2}-2 h\left(\gamma \alpha-\frac{2 \hat{k}_{2}}{\hat{k}_{1}} \bar{F}\left(1+\frac{\bar{F}}{\hat{k}_{1} \hat{r}}\right)\right)\left\|\hat{\sigma}_{k+1}\right\| \\
= & -\left(2 h \kappa_{1}-\|\hat{\epsilon}\|_{m}\right)\left\|\sigma_{k+1}+h \mathcal{A}_{k}^{-1} \xi_{k}\right\|^{2} \\
& -2 h\left(\gamma \alpha-\frac{2 \hat{k}_{2}}{\hat{k}_{1}} \bar{F}\left(1+\frac{\bar{F}}{\hat{k_{1} \hat{r}}}\right)\right)\left\|\sigma_{k+1}+h \mathcal{A}_{k}^{-1} \xi_{k}\right\| .
\end{aligned}
$$

Finally, from (46) and (40a) we can conclude that $\Delta V_{2}<0$ whenever $\left\|\sigma_{k+1}\right\|>$ $\left(\hat{r}+\frac{2 \bar{F}}{\hat{k}_{1}}\right) h$. Therefore, we obtain the ultimate boundedness of the solution of (31a), i.e., for any initial condition $\sigma_{0} \in \mathbb{R}^{n}$ we have that $\operatorname{dist}\left(\sigma_{k}, \sqrt{\frac{\hat{k}_{2}}{\hat{k}_{1}}}\left(\hat{r}+\frac{2 \bar{F}}{\hat{k}_{1}}\right) h \mathbb{B}_{n}\right) \rightarrow 0$ as $k \rightarrow \infty$.

Now we proceed with the proof of the finite-time convergence of $\hat{\sigma}_{k}$. Because of the ultimate boundedness of the solution of (31a) we have that there exists a finite number of steps $k^{*}$ such that $\left\|\sigma_{k}\right\| \leq 2 \sqrt{\frac{\hat{k}_{2}}{\hat{k}_{1}}}\left(\hat{r}+\frac{2 \bar{F}}{\hat{k}_{1}}\right) h$ for all $k \geq k^{*}$. Then, from (46) we have that

$$
\left\|\frac{\hat{M}_{k} \sigma_{k}}{h}\right\| \leq \frac{\hat{k}_{2}}{h}\left\|\sigma_{k}\right\| \leq 2 \hat{k}_{2} \sqrt{\frac{\hat{k}_{2}}{\hat{k}_{1}}}\left(\hat{r}+\frac{2 \bar{F}}{\hat{k}_{1}}\right) \leq \alpha \gamma,
$$

for all $k \geq k^{*}$. From Lemma 8 we obtain that $\hat{\sigma}_{k}$ reaches zero in at most $k^{*}+1$ steps. Moreover, $\hat{\sigma}_{k^{*}+n}=0$ for all $n \geq 1$, since the ball $2 \sqrt{\frac{\hat{k}_{2}}{\hat{k}_{1}}}\left(\hat{r}+\frac{2 \bar{F}}{\hat{k}_{1}}\right) h \mathbb{B}_{n}$ is positively 
invariant. Finally, since $\hat{\sigma}_{k^{*}+n}=0$ for all $n \geq 1$ it follows from (49) that

$$
\left\|\sigma_{k^{*}+n}\right\|=h\left\|\mathcal{B}_{k^{*}+n}^{-1} \xi_{k^{*}+n}\right\| \leq \frac{2 \bar{F}}{\hat{k}_{1}} h, \forall n \geq 1 .
$$

This concludes the proof.

Remark 8. Under the assumptions of Theorem 4, it becomes clear that $\sigma_{k}$ converges to a ball of radius $r_{\sigma}=\sqrt{\frac{\hat{k}_{2}}{\hat{k}_{1}}}\left(\hat{r}+\frac{2 \bar{F}}{\hat{k}_{1}}\right) h$, which implies boundedness of the state variable $\tilde{q}_{k}$. Recalling that $\Lambda$ and $h$ are such that Assumption 8 holds, the solution at the step $k$ is given by,

$$
\tilde{q}_{k}=(I-h \Lambda)^{k} \tilde{q}_{0}+h \sum_{n=0}^{k-1}(I-h \Lambda)^{(n+1)} \sigma_{k-n} .
$$

Hence, if $\sigma_{k}$ is bounded by $R_{\sigma}$ for all $k \in \mathbb{N}$ we have that

$$
\begin{aligned}
\lim _{k \rightarrow \infty}\left\|\tilde{q}_{k}\right\| & \leq h R_{\sigma} \sum_{n=0}^{\infty}\left\|(I-h \Lambda)^{n}\right\| \\
& \leq h R_{\sigma} \rho,
\end{aligned}
$$

for some $\rho>0$ [31, Theorem 22.11]. Therefore $\tilde{q}_{k}$ is also bounded for all $k \in \mathbb{N}$. Moreover, it converges to a ball of radius $h R_{\sigma} \rho$.

Corollary 2. Let the assumptions of Theorem 4 hold. Then in the case when there is no disturbance $(\xi \equiv 0)$, the origin of $(31)$ is globally finite-time Lyapunov stable, while $\tilde{q}_{k} \rightarrow 0$ asymptotically.

Proof. From (49) we have that $\sigma_{k+1}=\hat{\sigma}_{k+1}$ and the result follows since $\hat{\sigma}_{k}$ reaches the origin after a finite number of steps. The last statement follows by taking $R_{\sigma}=0$ in Remark 8.

Remark 9. It is worth to mention that the sampling period $h$ is uniform (i.e., it does not depend on the step $k$ ). Indeed, in view of Lemma 5 it becomes clear that $\hat{\epsilon}_{k}$ depends of both $\sigma_{k}$ and $\tilde{q}_{k}$, consequently the conditions in (40a) should be taken considering this dependency. Hence, all previous developments hold true if we consider the step length as a function of $k$ i.e., $h_{k}$, and we can conclude from Theorem 4 that $\sigma_{k}$ is at least bounded for all $k \in \mathbb{N}$. By the previous remark it follows that $\tilde{q}_{k}$ is bounded also for all $k \in \mathbb{N}$. Previous facts make $h_{k}$ uniformly bounded. Indeed, considering $\hat{\epsilon}_{k}$ as a function of $\tilde{q}_{k}$ and $\sigma_{k}$, taking the maximum $\max _{\left(\tilde{q}_{k}, \sigma_{k}\right)}\left\|\hat{\epsilon}_{k}\left(\tilde{q}_{k}, \sigma_{k}\right)\right\|$ (which exists since $\left(\sigma_{k}, \tilde{q}_{k}\right)$ its contained in a compact set of $\mathbb{R}^{2 n}$ for all $k \in \mathbb{N}$ ) allows us to compute $h$ independently of $k$.

Let us now consider the parametric uncertainty. In this case we see that the difference equation (31a) is equivalent to,

$$
\begin{aligned}
\hat{M}_{k} \sigma_{k+1}-\hat{M}_{k} \sigma_{k}+h \hat{C}_{k} \sigma_{k+1}+h K_{\sigma} \hat{\sigma}_{k+1}+h \xi_{k}+\left(M_{k}-\hat{M}_{k}\right) \sigma_{k+1} & \\
& -\left(M_{k}-\hat{M}_{k}\right) \sigma_{k}+h\left(C_{k}-\hat{C}_{k}\right) \sigma_{k+1}=-h \gamma \zeta_{k+1} .
\end{aligned}
$$

Defining two new perturbation terms associated with the parametric uncertainty as $\theta_{k}:=\left(M_{k}-\hat{M}_{k}\right) \dot{\sigma}_{k}$ and $\vartheta_{k}:=\left(C_{k}-\hat{C}_{k}\right) \sigma_{k+1}$, where $\sigma_{k+1}=\sigma_{k}+h \dot{\sigma}_{k}$ yields

$$
\hat{M}_{k} \sigma_{k+1}-\hat{M}_{k} \sigma_{k}+h \hat{C}_{k} \sigma_{k+1}+h K_{\sigma} \hat{\sigma}_{k+1}+h\left(\xi_{k}+\theta_{k}+\vartheta_{k}\right)=-h \gamma \zeta_{k+1} .
$$

The new two disturbance terms will add new constraints in both, the gain of the controller and the time step of the discrete-time scheme as is shown by the following theorem. 
Theorem 5 (Parametric uncertainty). Let Assumptions 1-8 hold. Consider the discrete time dynamical system (31). Assume that the parametric uncertainty is small enough such that there exists a $\hat{r}_{\sigma}>0$, considered fixed, and $h>0$ small enough such that $h \in\left(0, \min \left\{\delta^{*}, h^{*}\right\}\right]$, where $\delta^{*}$ is given by Lemma 7 and $h^{*}$ is specified below, and in addition, $\gamma$ and $\alpha$ satisfy

$$
\gamma \alpha>\max \left\{\frac{2 \hat{k}_{2}}{\hat{k}_{1}}\left(\bar{\beta}+f_{0} a_{0}\right)+\frac{2 \hat{k}_{2}}{\hat{k}_{1}^{2} \hat{r}_{\sigma}}\left(\bar{\beta}+f_{0} a_{0}\right)^{2}, 2 \hat{k}_{2} \sqrt{\frac{\hat{k}_{2}}{\hat{k}_{1}}}\left(\hat{r}_{\sigma}+\frac{2 \mathcal{F}}{\hat{k}_{1}}\right)\right\},
$$

where $a_{0}, a_{1}, \bar{\beta}, f_{0}$ and $\mathcal{F}$ are specified below in the proof. Then the origin of (31a) is semi-globally practically stable. Moreover, $\hat{\sigma}_{k}$ reaches the origin in a finite number of steps $k^{*}$, and $\hat{\sigma}_{k}=0$ for all $k \geq k^{*}+1$.

Proof. From (52) the analysis made in the proof of Theorem 4 can be achieved with the new perturbation term $\hat{\xi}_{k}=\xi_{k}+\vartheta_{k}+\theta_{k}$, but we must take special care since $\hat{\xi}_{k}$ is not uniformly bounded anymore. Consider the compact set $W:=\left\{w \in \mathbb{R}^{n} \mid w^{\top} \hat{M}_{0} w \leq R\right\}$ for some $R \in \mathbb{R}_{+}$. Hence for any $\sigma_{0} \in \mathbb{R}^{n}$ we can always find $R>0$ such that $\sigma_{0} \in W$. Moreover from Assumption 6 , there always exists $R_{\sigma}>0$ such that $W \subset R_{\sigma} \mathbb{B}_{n}$. So, following the same steps as in the proof of Theorem 4 we arrive at an inequality as in (51):

$$
\Delta V_{2} \leq-\left(2 h \kappa_{1}-\|\hat{\epsilon}\|_{m}\right)\left\|\hat{\sigma}_{k+1}\right\|^{2}-2 h\left(\gamma \alpha-\frac{2 \hat{k}_{2}}{\hat{k}_{1}}\left\|\hat{\xi}_{k}\right\|\right)\left\|\hat{\sigma}_{k+1}\right\|+4 h^{2} \frac{\hat{k}_{2}}{\hat{k}_{1}^{2}}\left\|\hat{\xi}_{k}\right\|^{2} .
$$

Now consider that $R$ is big enough such that $\left\|\hat{\sigma}_{k+1}\right\|>h \hat{r}_{\sigma}$ for all $\sigma_{k} \in \operatorname{bd}(W)$ where $\hat{r}_{\sigma}>0$ is a design variable considered fixed. Note that this is always possible since from (31c) we have that $\hat{\sigma}_{k+1}=\hat{\mathcal{A}}_{k}^{-1}\left(M_{k} \sigma_{k}-h \gamma \zeta_{k+1}\right)$ and the term $h \gamma \hat{\mathcal{A}}^{-1} \zeta_{k+1}$ is bounded, which implies that $\left\|\hat{\sigma}_{k+1}\right\| \rightarrow+\infty$ as $\left\|\sigma_{k}\right\| \rightarrow+\infty$. Hence, it follows that for every $\sigma_{k} \in \operatorname{bd}(W)$ :

$$
\Delta V_{2} \leq-\left(2 h \kappa_{1}-\|\hat{\epsilon}\|_{m}\right)\left\|\hat{\sigma}_{k+1}\right\|^{2}-2 h\left(\gamma \alpha-\frac{2 \hat{k}_{2}}{\hat{k}_{1}}\left\|\hat{\xi}_{k}\right\|-\frac{2 \hat{k}_{2}}{\hat{k}_{1}^{2} \hat{r}_{\sigma}}\left\|\hat{\xi}_{k}\right\|^{2}\right)\left\|\hat{\sigma}_{k+1}\right\| .
$$

The next step consists in finding appropriate bounds for the term $\left\|\hat{\xi}_{k}\right\|$ in the boundary of $W$. We have:

$$
\begin{aligned}
\left\|\hat{\xi}_{k}\right\| & \leq\left\|\xi_{k}\right\|+\left\|\vartheta_{k}\right\|+\left\|\theta_{k}\right\| \\
& \leq \bar{\beta}+\left\|M_{k}-\hat{M}_{k}\right\|_{m}\left\|\dot{\sigma}_{k}\right\|+\left\|C_{k}-\hat{C}_{k}\right\|_{m}\left\|\sigma_{k+1}\right\|,
\end{aligned}
$$

where $\bar{\beta}$ is an upper bound of $\beta\left(\sigma_{k}, \tilde{q}_{k}\right.$ ) (given in Proposition 5) as

$$
\bar{\beta}:=\max _{\left(\sigma_{k}, \tilde{q}_{k}\right) \in W \times \tilde{R} \mathbb{B}_{n}} \beta\left(\sigma_{k}, \tilde{q}_{k}\right),
$$

where $\tilde{R}=\tilde{R}\left(\sigma_{0}, \tilde{q}_{0}\right)$ is the radius of a closed ball such that $\tilde{q} \in \tilde{R} \mathbb{B}_{n}$ (we see that this is always possible in view of Remark 8). Recalling that $h \dot{\sigma}_{k}=\sigma_{k+1}-\sigma_{k}$, by (31a) it follows from Assumptions 1 and 6 that

$$
\begin{aligned}
\left\|\dot{\sigma}_{k}\right\| & =\left\|M_{k}^{-1}\left(C_{k} \sigma_{k+1}-K_{\sigma} \hat{\mathcal{A}}_{k}^{-1} \hat{M}_{k} \sigma_{k}-\left(I-h K_{\sigma} \hat{\mathcal{A}}_{k}^{-1}\right) \gamma \zeta_{k+1}+\xi_{k}\right)\right\| \\
& \leq \frac{1}{k_{1}}\left[k_{C}\left\|\dot{q}_{k}\right\|\left\|\sigma_{k+1}\right\|+\frac{\kappa_{2} \hat{k}_{2}}{\hat{k}_{1}} R_{\sigma}+\left(1+h \frac{\kappa_{2}}{\hat{k}_{1}}\right) \gamma R_{\zeta}+\bar{\beta}\right]
\end{aligned}
$$


where $\kappa_{2}$ is the maximum eigenvalue of $K_{\sigma}$ and $R_{\zeta}$ is an upper-bound of $\zeta_{k+1}$. Thus, from (57) we obtain

$$
\left\|\hat{\xi}_{k}\right\| \leq\left(1+a_{1}\right) \bar{\beta}+a_{1} \frac{\kappa_{2} k_{2}}{\hat{k}_{1}} R_{\sigma}+\left(1+h \frac{\kappa_{2}}{\hat{k}_{1}}\right) a_{1} \gamma R_{\zeta}+\left(a_{1} k_{C}\left\|\dot{q}_{k}\right\|+a_{0}\right)\left\|\sigma_{k+1}\right\| .
$$

where

$$
\begin{aligned}
& a_{0}:=\left\|C_{k}-\hat{C}_{k}\right\|_{m} \\
& a_{1}:=\frac{\left\|M_{k}-\hat{M}_{k}\right\|_{m}}{k_{1}} .
\end{aligned}
$$

Once again from (31a) it follows that

$$
\begin{aligned}
\left\|\sigma_{k+1}\right\| & =\left\|\mathcal{B}_{k}^{-1}\left(\left(M_{k}-h K_{\sigma} \hat{\mathcal{A}}_{k}^{-1} \hat{M}_{k}\right) \sigma_{k}-\left(1-h K_{\sigma} \hat{\mathcal{A}}_{k}^{-1}\right) h \gamma \zeta_{k+1}-h \xi_{k}\right)\right\| \\
& \leq \frac{2}{k_{1}}\left(\left(k_{2}+h \frac{\kappa_{2} \hat{k}_{2}}{\hat{k}_{1}}\right) R_{\sigma}+\left(1+h \frac{\kappa_{2}}{\hat{k}_{1}}\right) h \gamma R_{\zeta}+h \bar{\beta}\right),
\end{aligned}
$$

where we made use of the analog of Proposition 6 for $\mathcal{B}_{k}^{-1}$ (see Remark 7). After some algebraic operations, the substitution of (61) into (58) results in

$$
\left\|\hat{\xi}_{k}\right\| \leq b_{0}+b_{1} h+b_{2} h^{2}=: \mathcal{F}
$$

where each $b_{i}>0$ is given by

$$
\begin{aligned}
b_{0} & :=\bar{\beta}+2 \frac{\kappa_{2}}{k_{1}} a_{0} R_{\sigma}+\left(\bar{\beta}+\gamma R_{\zeta}+\left(\frac{\hat{k}_{2}}{\hat{k}_{1}} \kappa_{2}+2 \frac{k_{2}}{k_{1}} k_{C} R_{q}\right) R_{\sigma}\right) a_{1}, \\
b_{1} & :=\frac{\kappa_{2}}{\hat{k}_{1}} \gamma R_{\zeta} a_{1}+\left(\bar{\beta}+\gamma R_{\zeta}+2 \frac{\kappa_{2} k_{2}}{k_{1} \hat{k}_{1}} R_{\sigma}\right)\left(a_{0}+k_{C} R_{q} a_{1}\right) \\
b_{2} & :=\frac{\kappa_{2}}{\hat{k}_{1}} \gamma R_{\zeta}\left(a_{0}+k_{C} R_{q} a_{1}\right)
\end{aligned}
$$

and $R_{q}<+\infty$ corresponds to an upper-bound of $\dot{q}_{k}$ (which exists because in the boundary of $W$ both $\sigma_{k}$ and $\tilde{q}_{k}$ are bounded). Hence, from (55) and (62) it is clear that we obtain the positive invariance of $W$ whenever

$$
g(h):=-\gamma \alpha+\frac{2 \hat{k}_{2}}{\hat{k}_{1}}\left(b_{0}+b_{1} h+b_{2} h^{2}\right)+\frac{2 \hat{k}_{2}}{\hat{k}_{1}^{2} \hat{r}_{\sigma}}\left(b_{0}+b_{1} h+b_{2} h^{2}\right)^{2}<0 .
$$

After some inspection, condition (64) can be written in the form $g(h):=d_{4} h^{4}+d_{3} h^{3}+$ $d_{2} h^{2}+d_{1} h+d_{0}<0$, where $d_{i}>0$ for $i=1, \ldots, 4$. It follows that if $d_{0}<0$, then by the continuity of $g$ we have that there exists $h^{*}>0$ such that $g(h)<0$ for all $h \in\left(0, h^{*}\right]$. From (64) and (63a) it follows that $d_{0}$ is given by,

$$
\begin{aligned}
d_{0}= & -\gamma \alpha+\frac{2 \hat{k}_{2}}{\hat{k}_{1}} b_{0}+\frac{2 \hat{k}_{2}}{\hat{k}_{1}^{2} \hat{r}_{\sigma}} b_{0}^{2} \\
= & -\gamma \alpha+\frac{2 \hat{k}_{2}}{\hat{k}_{1}}\left(\bar{\beta}+f_{0} a_{0}+f_{1} a_{1}\right)+\frac{2 \hat{k}_{2}}{\hat{k}_{1}^{2} \hat{r}_{\sigma}}\left(\bar{\beta}+f_{0} a_{0}+f_{1} a_{1}\right)^{2} \\
= & -\gamma \alpha+\frac{2 \hat{k}_{2}}{\hat{k}_{1}}\left(\bar{\beta}+f_{0} a_{0}\right)+\frac{2 \hat{k}_{2}}{\hat{k}_{1}^{2} \hat{r}_{\sigma}}\left(\bar{\beta}+f_{0} a_{0}\right)^{2}+2 \frac{\hat{k}_{2}}{\hat{k}_{1}}\left(1+\frac{2}{\hat{k}_{1} \hat{r}_{\sigma}}\left(\bar{\beta}+f_{0} a_{0}\right)\right) f_{1} a_{1} \\
& +\frac{2 \hat{k}_{2}}{\hat{k}_{1}^{2} \hat{r}_{\sigma}} f_{1}^{2} a_{1}^{2},
\end{aligned}
$$


where $f_{0}:=2 \frac{\kappa_{2}}{k_{1}} R_{\sigma}$ and $f_{1}:=\bar{\beta}+\gamma R_{\zeta}+\left(\frac{\hat{k}_{2}}{\hat{k}_{1}} \kappa_{2}+2 \frac{k_{2}}{k_{1}} k_{C} R_{q}\right) R_{\sigma}$. Following the same reasoning, we have that $d_{0}$ can be viewed as a second order polynomial in $a_{1}$ with independent term given by

$$
-\gamma \alpha+\frac{2 \hat{k}_{2}}{\hat{k}_{1}}\left(\bar{\beta}+f_{0} a_{0}\right)+\frac{2 \hat{k}_{2}}{\hat{k}_{1}^{2} \hat{r}_{\sigma}}\left(\bar{\beta}+f_{0} a_{0}\right)^{2},
$$

which is negative in view of $(53)$. Then, $d_{0}$ will be negative whenever the parametric uncertainty is small enough, i.e., since the independent term in the polynomial $d_{0}\left(a_{1}\right)$ is negative there exists an interval $\left[0, a_{1}^{*}\right]$ such that $d_{0}<0$ for all $a_{1} \in\left[0, a_{1}^{*}\right]$. This last fact implies that there exists an interval $\left(0, h^{*}\right]$ such that for any $h \in\left(0, h^{*}\right]$ we have that $\Delta V_{2}<0$, which proves the positive invariance of $W$.

Notice that in the case without parametric uncertainty we have $a_{1}=a_{0}=0$ and from (62)-(63) it becomes clear that the polynomial $g(h)$ reduces to

$$
g(h)=-\gamma \alpha+2 \frac{\hat{k}_{2}}{\hat{k}_{1}} \bar{\beta}+2 \frac{\hat{k}_{2}}{\hat{k}_{1}^{2} \hat{r}_{\sigma}} \bar{\beta}^{2},
$$

which is strictly negative in the light of (46) with $\hat{r}=\hat{r}_{\sigma}$ and $\bar{\beta}=\bar{F}$. Let us now pass to the last part of the theorem. Assume that we start at $k=0$ with an initial condition $\sigma_{0} \in \mathbb{R}^{n}$. Then, there exists $R>0$ such that $\sigma_{0} \in W$. Moreover, there exists $R_{\sigma}>0$ such that $W \subset R_{\sigma} \mathbb{B}_{n}$. Since $W$ is invariant, it follows that $\left\|\xi_{k}\right\|$ is bounded by $\bar{\beta}$ for all $k \in \mathbb{N}$. Previous statements imply that that the bound (62) is still valid for all $k \in \mathbb{N}$. Then from (54) and considering that $\left\|\sigma_{k+1}\right\|>\left(\hat{r}_{\sigma}+2 \frac{\mathcal{F}}{\hat{k}_{1}}\right) h$, where $\hat{r}_{\sigma}>0$ is fixed and $\mathcal{F}$ is defined in (62), (notice that as in the proof of Theorem 4 the constraint $\left\|\sigma_{k+1}\right\|>\left(\hat{r}_{\sigma}+2 \frac{\mathcal{F}}{\hat{k}_{1}}\right) h$ implies $\left.\left\|\hat{\sigma}_{k+1}\right\| \geq \hat{r}_{\sigma} h\right)$, we have that

$$
\Delta V_{2} \leq-\left(2 h \kappa_{1}-\|\hat{\epsilon}\|_{m}\right)\left\|\hat{\sigma}_{k+1}\right\|^{2}-2 h\left(\gamma \alpha-\frac{\hat{k}_{2}}{\hat{k}_{1}}\left\|\hat{\xi}_{k}\right\|-\frac{\hat{k}_{2}}{2 \hat{k}_{1}^{2} \hat{r}_{\sigma}}\left\|\hat{\xi}_{k}\right\|^{2}\right)\left\|\hat{\sigma}_{k+1}\right\|
$$

For semi-global practical stability we need to prove that the term within parenthesis that pre-multiplies $\left\|\hat{\sigma}_{k+1}\right\|$ is negative, but in the first part of the proof we have proved that

$$
-\gamma \alpha+2 \frac{\hat{k}_{2}}{\hat{k}_{1}}\left\|\hat{\xi}_{k}\right\|+2 \frac{\hat{k}_{2}}{\hat{k}_{1}^{2} \hat{r}_{\sigma}}\left\|\hat{\xi}_{k}\right\|^{2}<g(h)<0,
$$

whenever $a_{1}$ and $h$ are small enough. Therefore, $\Delta V_{2}<0$ for all $\left\|\sigma_{k+1}\right\|>\left(\hat{r}_{\sigma}+2 \frac{\mathcal{F}}{\hat{k}_{1}}\right) h$. The proof for the finite-time convergence mimics the corresponding part of the proof of Theorem 4 and for that reason we omit it here. This concludes the proof.

\subsection{Convergence of the discrete-time solutions}

In this section we show that the implicit Euler method used for the design of the discretetime controller provides a well-posed approximation of the continuous-time system, in the sense that the solutions of the discrete scheme (31) defined on an interval $[0, T]$, $T<+\infty$, converge to solutions of

$$
\left\{\begin{array}{l}
M(q(t)) \dot{\sigma}(t)+C(q(t), \dot{q}(t)) \sigma(t)+K_{\sigma} \sigma(t)+\xi(t, \sigma(t), \tilde{q}(t))=-\gamma \zeta(t), \\
\zeta(t) \in \partial \Phi(\sigma(t)) \\
\dot{\tilde{q}}(t)=\sigma(t)-\Lambda \tilde{q}(t) \\
\sigma(0)=\sigma_{0}, \tilde{q}(0)=\tilde{q}_{0}
\end{array}\right.
$$


To this end we form the following piecewise-linear approximations

$$
\begin{aligned}
\sigma_{h}(t) & :=\sigma_{k+1}+\frac{t_{k+1}-t}{h}\left(\sigma_{k}-\sigma_{k+1}\right), \text { for all } t \in\left[t_{k}, t_{k+1}\right) \\
q_{h}(t) & :=q_{k+1}+\frac{t_{k+1}-t}{h}\left(q_{k}-q_{k+1}\right), \text { for all } t \in\left[t_{k}, t_{k+1}\right) . \\
\tilde{q}_{h}(t) & :=\tilde{q}_{k+1}+\frac{t_{k+1}-t}{h}\left(\tilde{q}_{k}-\tilde{q}_{k+1}\right), \text { for all } t \in\left[t_{k}, t_{k+1}\right) .
\end{aligned}
$$

where $h=t_{k+1}-t_{k}$ and $T=N h$ for some $N \in \mathbb{N}$. From (66b) and (66c) we see that $\tilde{q}_{h}(t)=q_{h}(t)-q_{h}^{\mathrm{d}}(t)$, where $q_{h}^{\mathrm{d}}$ is the piecewise-linear approximation to $q^{d}$. We also define the following step-functions:

$$
\begin{aligned}
\sigma_{h}^{*}(t) & :=\sigma_{k+1}, \text { for all } t \in\left[t_{k}, t_{k+1}\right) . \\
\hat{\sigma}_{h}^{*}(t) & :=\hat{\sigma}_{k+1}, \text { for all } t \in\left[t_{k}, t_{k+1}\right) . \\
q_{h}^{*}(t) & :=q_{k}, \text { for all } t \in\left[t_{k}, t_{k+1}\right) . \\
\xi_{h}(t) & :=\xi\left(t_{k}, \sigma_{k}, \tilde{q}_{k}\right), \text { for all } t \in\left[t_{k}, t_{k+1}\right) .
\end{aligned}
$$

By construction it becomes easy to prove, in view of Theorem 5 , that given a set $W$, then for any $\sigma_{0} \in W, \sigma_{h} \in C\left([0, T] ; \mathbb{R}^{n}\right)$ and $\tilde{q}_{h} \in C\left([0, T] ; \mathbb{R}^{n}\right)$, where $C\left([0, T] ; \mathbb{R}^{n}\right)$ refers to the Banach space of all continuous functions with domain $[0, T]$, range in $\mathbb{R}^{n}$ and norm

$$
\|f\|_{C}=\sup _{t \in[0, T]}\|f(t)\| .
$$

Moreover, again as a consequence of Theorem 5, (31) and (38), we have that $\dot{\sigma}_{h} \in$ $L^{\infty}\left([0, T] ; \mathbb{R}^{n}\right)$ and $\dot{\tilde{q}}_{h} \in L^{\infty}\left([0, T] ; \mathbb{R}^{n}\right)$. Since the sequences $\left\{\sigma_{h}\right\}$ and $\left\{\tilde{q}_{h}\right\}$ are continuous with uniformly bounded derivatives almost everywhere, it follows that both families are equicontinuous. Hence, by a direct application of the Arzelà-Ascoli Theorem [24, Theorem 1.3.8], we have that $\left\{\sigma_{h}\right\}$ and $\left\{\tilde{q}_{h}\right\}$ remain in a compact subset of $C\left([0, T] ; \mathbb{R}^{n}\right)$ and therefore there exist subsequences (still denoted by) $\left\{\sigma_{h}\right\}$ and $\left\{\tilde{q}_{h}\right\}$ for which

$$
\begin{gathered}
\sigma_{h} \rightarrow \sigma \text { as } h \downarrow 0, \\
\tilde{q}_{h} \rightarrow \tilde{q} \text { as } h \downarrow 0,
\end{gathered}
$$

uniformly in $C\left([0, T] ; \mathbb{R}^{n}\right)$ and for some functions $\sigma$ and $\tilde{q}$. In addition we have

$$
\begin{aligned}
\left\|\sigma_{h}(t)-\sigma_{h}^{*}(t)\right\|_{L^{2}\left([0, T] ; \mathbb{R}^{n}\right)}^{2} & =\sum_{k=0}^{N-1} \int_{t_{k}}^{t_{k+1}}\left(t_{k+1}-t\right)^{2}\left\|\dot{\sigma}_{k}\right\|^{2} d t \\
& \leq C_{1}^{2} \sum_{k=0}^{N-1} \int_{t_{k}}^{t_{k+1}}\left(t_{k+1}-t\right)^{2} d t \\
& \leq C_{1}^{2} \sum_{k=0}^{N-1} \frac{1}{3}\left(t_{k+1}-t_{k}\right)=\frac{C_{1}^{2} T h^{2}}{3}
\end{aligned}
$$

where $\left\|\dot{\sigma}_{k}\right\| \leq C_{1}$, for all $k \in \mathbb{N}$. Hence, it follows from (70) that $\sigma_{h}^{*} \rightarrow \sigma$ as $h \downarrow 0$ strongly in $L^{2}\left([0, T] ; \mathbb{R}^{n}\right)$. From (52) together with (31c) the following equality holds

$$
\sigma_{k+1}=\hat{\sigma}_{k+1}+h \hat{\mathcal{B}}_{k}^{-1} \hat{\xi}_{k}
$$

and consequently, making use of (62), we obtain

$$
\left\|\sigma_{h}^{*}(t)-\hat{\sigma}_{h}^{*}(t)\right\| \leq h \frac{2}{\hat{k}_{1}}\left\|\hat{\xi}_{k}\right\| \leq h \frac{2}{\hat{k}_{1}}\left(b_{0}+b_{1} h+b_{2} h^{2}\right) .
$$


Hence, it follows that $\hat{\sigma}_{h}^{*} \rightarrow \sigma$ as $h \downarrow 0$ strongly in $L^{2}\left([0, T] ; \mathbb{R}^{n}\right)$. On the other hand, we have that $\dot{\sigma}_{h} \in L^{\infty}\left([0, T] ; \mathbb{R}^{n}\right)$, then from the Banach-Alaoglu Theorem [24, Theorem 2.4.3] it follows that $\dot{\sigma}_{h} \rightarrow s$ in the weak* topology, i.e.,

$$
\lim _{h \downarrow 0} \int_{0}^{T}\left\langle\dot{\sigma}_{h}(t)-s(t), \varphi(t)\right\rangle d t=0, \text { for all } \varphi \in L^{1}\left([0, T] ; \mathbb{R}^{n}\right),
$$

Since the domain is bounded, it follows that $L^{1}\left([0, T] ; \mathbb{R}^{n}\right) \subseteq L^{2}\left([0, T] ; \mathbb{R}^{n}\right)$ and consequently we have that $\dot{\sigma}_{h}$ converges weakly in $L^{2}\left([0, T], \mathbb{R}^{n}\right)$. Moreover, since $\sigma_{h}(t)=$ $\sigma_{h}(0)+\int_{0}^{T} s(t) d t$, we infer that $s=\dot{\sigma}$ almost everywhere. Summarizing we have

$$
\begin{aligned}
\sigma_{h} & \rightarrow \sigma \text { strongly in } L^{2}\left([0, T], \mathbb{R}^{n}\right) \\
\tilde{q}_{h} & \rightarrow \tilde{q} \text { strongly in } L^{2}\left([0, T], \mathbb{R}^{n}\right) \\
\dot{\tilde{q}}_{h} & \rightarrow \dot{\tilde{q}} \text { strongly in } L^{2}\left([0, T], \mathbb{R}^{n}\right) \\
q_{h} & \rightarrow q \text { strongly in } L^{2}\left([0, T], \mathbb{R}^{n}\right) \\
\dot{q}_{h} & \rightarrow \dot{q} \text { strongly in } L^{2}\left([0, T], \mathbb{R}^{n}\right) \\
\dot{\sigma}_{h} & \rightarrow \dot{\sigma} \text { weakly in } L^{2}\left([0, T], \mathbb{R}^{n}\right) .
\end{aligned}
$$

From Assumptions 3 and 5 it follows that $C\left(q_{k}, \dot{q}_{k}\right) \sigma_{k+1} \rightarrow C(q, \dot{q}) \sigma$ and $G\left(q_{k}\right) \rightarrow G(q)$ both strongly in $L^{2}\left([0, T] ; \mathbb{R}^{n}\right)$. An additional assumption is considered with respect to the disturbance $F(t, q, \dot{q})$ in (8) as follows:

Assumption 9. The derivative of $F(\cdot, q, \dot{q})$ maps bounded sets of $L^{2}\left([0, T] ; \mathbb{R}^{n}\right)$ into bounded sets of $L^{2}\left([0, T] ; \mathbb{R}^{n}\right)$, i.e.,

$$
\sup \left\{\left\|\frac{\partial F}{\partial t}(\cdot, q, \dot{q})\right\|_{L^{2}\left([0, T] ; \mathbb{R}^{n}\right)}:\|q\|_{L^{2}\left([0, T] ; \mathbb{R}^{n}\right)},\|\dot{q}\|_{L^{2}\left([0, T] ; \mathbb{R}^{n}\right)} \leq R\right\}<+\infty .
$$

Assumption 9 guarantees that $F_{h} \rightarrow F(\cdot, q, \dot{q})$ strongly in $L^{2}\left([0, T] ; \mathbb{R}^{n}\right)$, see e.g., $[5,7]$. This implies in turn the strong convergence of $\xi_{h}$ to $\xi(\cdot, \sigma, \tilde{q})$ in $L^{2}\left([0, T] ; \mathbb{R}^{n}\right)$. Until this point it only remains to prove the weak convergence of the term $M\left(q_{h}^{*}\right) \dot{\sigma}_{h} \in L^{2}\left([0, T] ; \mathbb{R}^{n}\right)$ to $M(q) \dot{\sigma}$. Let $\varphi \in L^{2}\left([0, T] ; \mathbb{R}^{n}\right)$,

$$
\begin{aligned}
\mid \int_{0}^{T}\left\langle M\left(q_{h}^{*}(t)\right) \dot{\sigma}_{h}(t)\right. & -M(q(t)) \dot{\sigma}(t), \varphi(t)\rangle d t \mid \\
\leq & \left|\int_{0}^{T}\left\langle\left(M\left(q_{h}^{*}(t)\right)-M(q(t))\right) \dot{\sigma}_{h}(t), \varphi(t)\right\rangle d t\right| \\
& +\left|\int_{0}^{T}\left\langle M(q(t))\left(\dot{\sigma}_{h}(t)-\dot{\sigma}(t)\right), \varphi(t)\right\rangle d t\right| \\
\leq & k_{3} \int_{0}^{T}\left\|q_{h}^{*}(t)-q(t)\right\|\left\|\dot{\sigma}_{h}(t)\right\|\|\varphi(t)\| d t \\
& +\int_{0}^{T}\left\langle\dot{\sigma}_{h}(t)-\dot{\sigma}(t), M(q(t)) \varphi(t)\right\rangle d t .
\end{aligned}
$$

Hence, from (73) and (74) it follows that,

$$
M\left(q_{h}^{*}\right) \dot{\sigma}_{h}+C\left(q_{h}^{*}, \dot{q}_{h}\right) \sigma_{h}^{*}+K_{\sigma} \hat{\sigma}_{h}^{*}+\xi_{h} \rightarrow M(q) \dot{\sigma}+C(q, \dot{q}) \sigma+K_{\sigma} \sigma+\xi,
$$

weakly in $L^{2}\left([0, T] ; \mathbb{R}^{n}\right)$. Finally, since the operator $\partial \Phi$ is a maximal monotone operator, a direct application of Proposition 2 in [6, Chapter 3.1] gives the convergence of solutions of (31) towards solutions of (65). In other words, we have proved that the chosen timediscretization in Section 6.1 is a good approximation of the continuous-time dynamics. This is an important conclusion since the discrete-time controller is designed from an approximation of the continuous-time plant. 


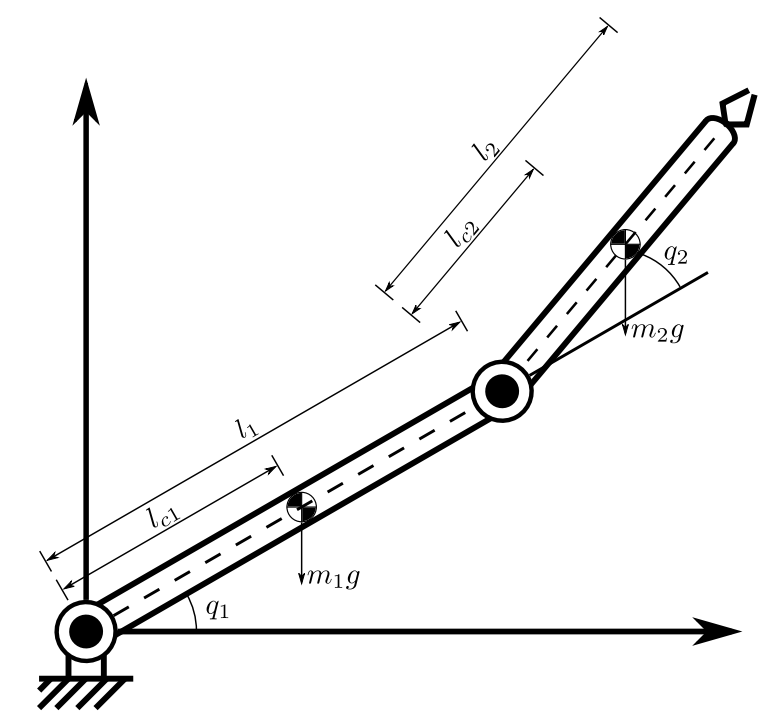

Figure 2: Diagram of a two-link planar elbow manipulator.

\section{Examples}

In this section we present some examples of application of the control law developed in Section 6. Two scenarios are considered. First we apply the control law described by (28)-(29) to the discrete-time plant (23) for several values of the time step $h>0$. After that a more realistic setting is proposed, i.e., we consider the continuous-time plant (8) and we apply the stepwise controller obtained from (28)-(29). In the last case the values of the state of the plant are measured through a zero-order-hold mechanism, creating a new perturbation due to the fact that the discretization from which the discrete-time input is designed, is not exact. The robustness of the discrete-time controller with respect to this perturbation is tested numerically.

\subsection{Control in the discrete/discrete setting}

Consider the two-link planar elbow manipulator depicted in Figure 2. Its dynamics is given by (8) with

$$
\begin{aligned}
M(q) & =\left[\begin{array}{cc}
m_{1} l_{c 1}^{2}+m_{2} a+I_{1}+I_{2} & m_{2}\left(l_{c 2}^{2}+l_{1} l_{c 2} \cos \left(q_{2}\right)\right)+I_{2} \\
m_{2}\left(l_{c 2}^{2}+l_{1} l_{c 2} \cos \left(q_{2}\right)\right)+I_{2} & m_{2} l_{c 2}^{2}+I_{2}
\end{array}\right], \\
C(q, \dot{q}) & =-m_{2} l_{1} l_{c 2} \sin \left(q_{2}\right)\left[\begin{array}{cc}
\dot{q}_{2} & \dot{q}_{1}+\dot{q}_{2} \\
-\dot{q}_{1} & 0
\end{array}\right], \\
G(q) & =\left[\begin{array}{c}
\left(m_{1} l_{c 1}+m_{2} l_{1}\right) g \cos \left(q_{1}\right)+m_{2} l_{c 2} g \cos \left(q_{1}+q_{2}\right) \\
m_{2} l_{c 2} \cos \left(q_{1}+q_{2}\right)
\end{array}\right],
\end{aligned}
$$

where $m_{i}$ represents the mass of the $i t h-$ link, $l_{i}$ and $l_{c i}$, are the length of the $i t h$-link and the distance from the base of the $i t h$-link to its center of mass respectively, $I_{i}$ is the inertia moment of the $i t h-$ link, for $i=1,2, g=9.81 \mathrm{~m} / \mathrm{s}^{2}$ is the gravitational constant and $a:=l_{1}^{2}+l_{c 2}^{2}+2 l_{1} l_{c 2}\left(l_{c 2}+\cos \left(q_{2}\right)\right)$. The parameters of the real plant are as follows:

\begin{tabular}{|l|l||l|l|}
\hline$m_{1}$ & $1.5 \mathrm{~kg}$. & $m_{2}$ & $1.0 \mathrm{~kg}$. \\
\hline$l_{1}$ & $0.4 \mathrm{~m}$ & $l_{2}$ & $0.3 \mathrm{~m}$ \\
\hline$l_{c 1}$ & $0.2 \mathrm{~m}$ & $l_{c 2}$ & $0.2 \mathrm{~m}$ \\
\hline$I_{1}$ & 0.08 & $I_{2}$ & 0.03 \\
\hline
\end{tabular}


In this example, our control objective consists in the tracking control of the following trajectory:

$$
q_{\mathrm{d}}(t)=\frac{\pi}{2}\left[\begin{array}{c}
\sin (t)+1 \\
-\cos (t)
\end{array}\right], \dot{q}_{\mathrm{d}}(t)=\frac{\pi}{2}\left[\begin{array}{c}
\cos (t) \\
\sin (t)
\end{array}\right], \ddot{q}_{\mathrm{d}}(t)=\frac{\pi}{2}\left[\begin{array}{c}
-\sin (t) \\
\cos (t)
\end{array}\right] .
$$

Moreover, we consider that the system is subject to a disturbance given as:

$$
F(t, q, \dot{q})=0.25\left[\begin{array}{c}
\cos (\pi t) \sin (t) \\
0.5 \sin (\sqrt{2} t) \sin (t / 3) \cos (t)
\end{array}\right]+0.5\left[\begin{array}{c}
\tanh \left(\dot{q}_{1}\right) \\
\tanh \left(q_{2}\right) \cos \left(q_{1}+q_{2}\right)
\end{array}\right],
$$

whereas the nominal parameters are:

\begin{tabular}{|l|l||l|l|}
\hline$\hat{m}_{1}$ & $1.6 \mathrm{~kg}$. & $\hat{m}_{2}$ & $0.8 \mathrm{~kg}$. \\
\hline$\hat{l}_{1}$ & $0.4 \mathrm{~m}$ & $\hat{l}_{2}$ & $0.3 \mathrm{~m}$ \\
\hline$\hat{l}_{c 1}$ & $0.25 \mathrm{~m}$ & $\hat{l}_{c 2}$ & $0.15 \mathrm{~m}$ \\
\hline$\hat{I}_{1}$ & 0.0853 & $\hat{I}_{2}$ & 0.0240 \\
\hline
\end{tabular}

In this case the gains of the controller are set as:

$$
K_{\sigma}=2\left[\begin{array}{cc}
5 & -4 \\
-4 & 5
\end{array}\right], \Lambda=\left[\begin{array}{cc}
5 & -0.5 \\
-0.5 & 8
\end{array}\right], \gamma=3, \alpha=1
$$

In all this section we set $\Phi(x)=\alpha\|x\|_{1}$. With this choice of $\Phi$, the algorithm described by (38) for the calculation of the control selection is implemented for each $k \in \mathbb{N}$ using the successive approximations method as follows:

1. Set $\mu>0$ small enough such that (39) holds.

2. Set $j=0$ and set $x^{0} \in \mathbb{R}^{n}$.

3. Compute $x^{j+1}$ as

$$
\begin{aligned}
v^{j} & =\left(I-\mu \hat{\mathcal{A}}_{k}\right) x^{j}+\mu \hat{M}_{k} \sigma_{k}, \\
x^{j+1} & =v^{j}-\mu \operatorname{Proj}_{[-c, c]^{n}}\left(\frac{v^{j}}{\mu}\right),
\end{aligned}
$$

where $c=h \gamma \alpha$, and the set $[-c, c]^{n}$ represents the $n$-cube in $\mathbb{R}^{n}$ centered in the origin with edge length equal to $2 c$, i.e., $[-c, c]^{n}:=\left\{w \in \mathbb{R}^{n} \mid w_{i} \in[-c, c]\right.$, for all $i=$ $1, \ldots, n\}$.

4. If $\left\|x^{j+1}-x^{j}\right\|>\varepsilon$, then increase $j$ and go to step 3 . Else, set $\hat{\sigma}_{k+1}=x^{j+1}$ and stop.

In the previous algorithm, the variable $\varepsilon$ represents the precision of the algorithm which in all the simulations is set to $10^{-9}$. The sliding variable and control input of the closedloop system (23) with control law (28)-(29) are shown in Figures $3-5$ for the time steps $h=10^{-1} s, h=10^{-2} s$ and $h=10^{-3} s$ respectively. In all the simulations the initial data are given by $\left[q_{0}^{\top}, \dot{q}_{0}^{\top}\right]=[\pi / 3,-\pi / 4, \pi / 3, \pi / 8]^{\top}$. The plots show how the tracking error decreases as $h$ decreases (since $\sigma_{k} \rightarrow 0$ as $h \downarrow 0$ ), as expected. It is also worth to mention that the control input magnitude is independent of the sampling-time $h$ in the discrete-time sliding phase, a property of the implicit discretization method noticed in $[17,18,19,40]$. Moreover, in all of the three cases there is no chattering present at all, neither in the input nor in the output. Finally, the nominal sliding variable $\hat{\sigma}$ reaches zero in a finite number of steps and it is maintained at zero (i.e., the system is in the discrete-time sliding phase [19]). 

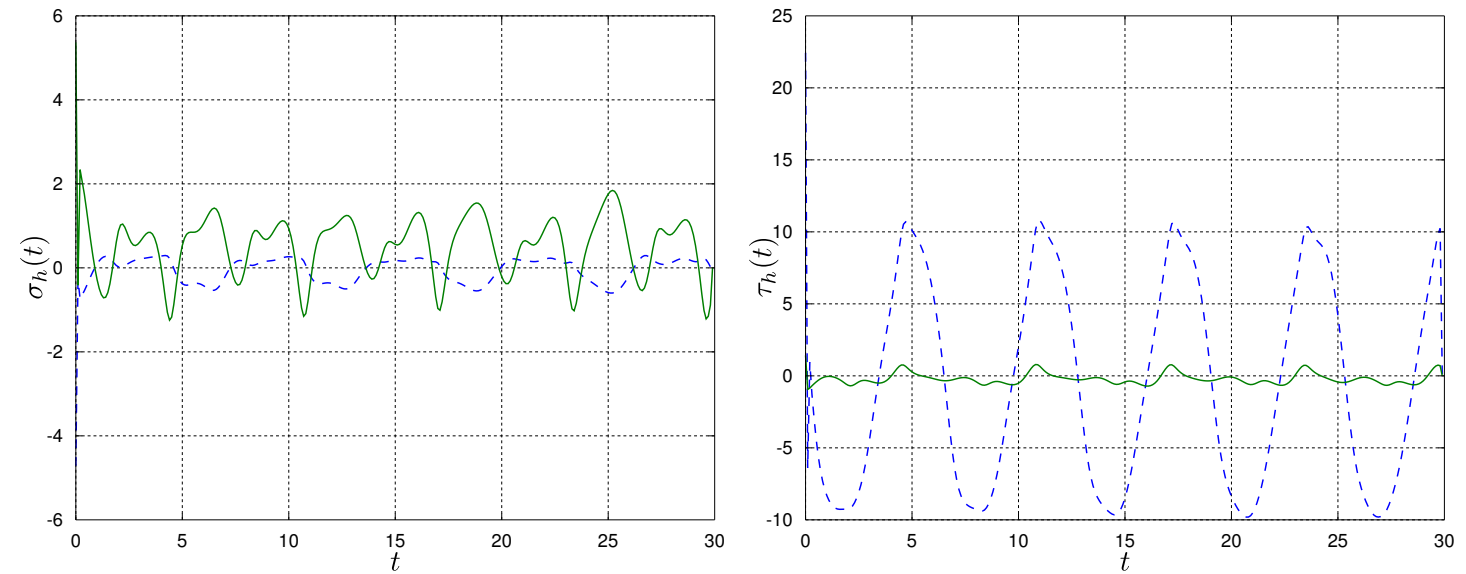

Figure 3: Evolution of the sliding variable $\sigma_{h}$ (left) and the input $\tau_{h}$ (right) for the closed-loop system (23) (28) (29) with sampling time $h=10^{-1} s$.
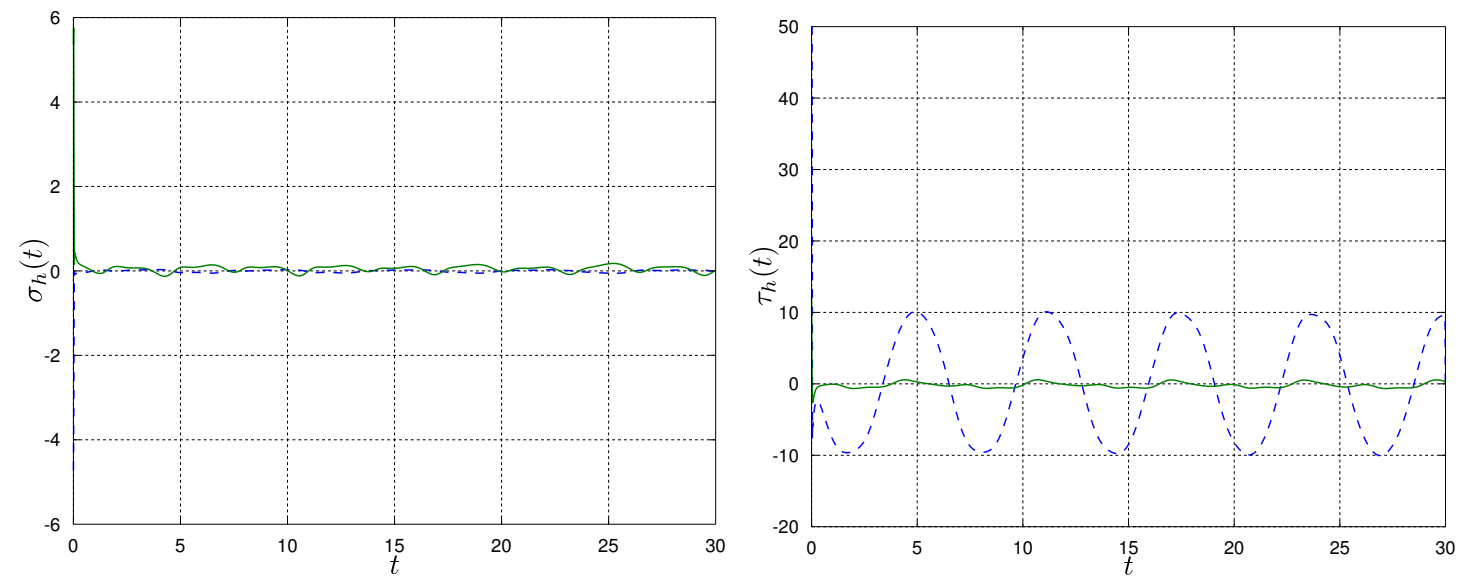

Figure 4: Evolution of the sliding variable $\sigma_{h}$ (left) and the input $\tau_{h}$ (right) for the closed-loop system (23) (28) (29) with sampling time $h=10^{-2} s$.
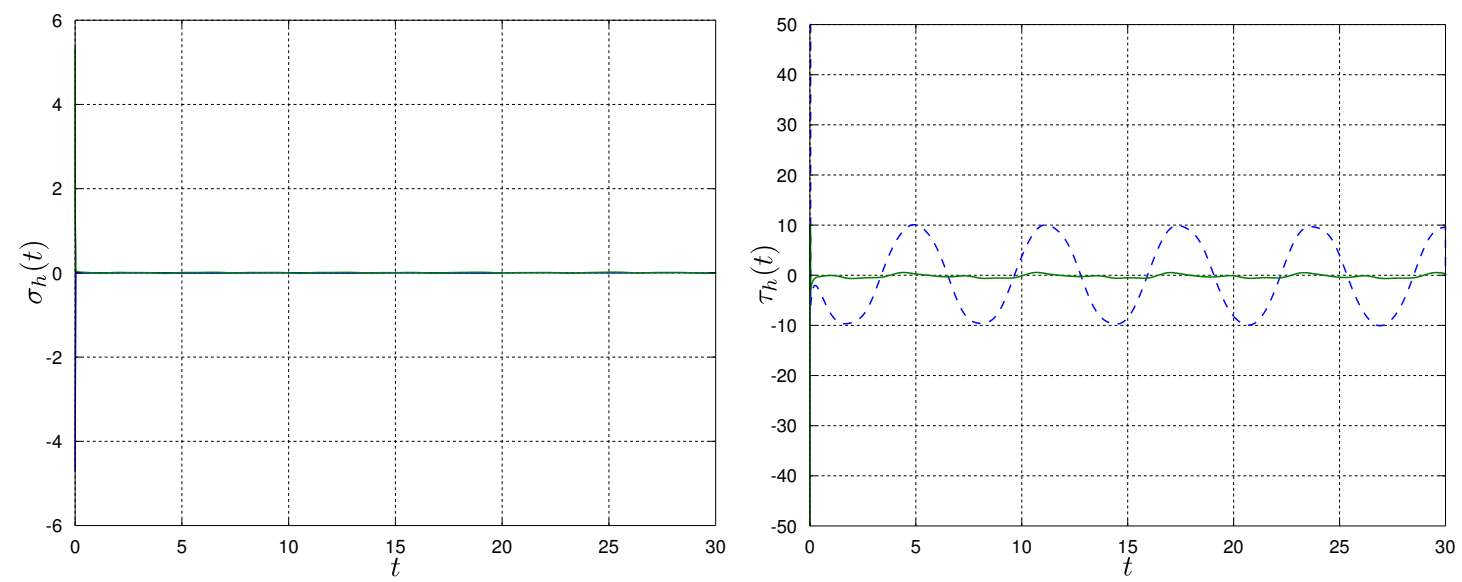

Figure 5: Evolution of the sliding variable $\sigma_{h}$ (left) and the input $\tau_{h}$ (right) for the closed-loop system (23) (28) (29) with sampling time $h=10^{-3} s$. 

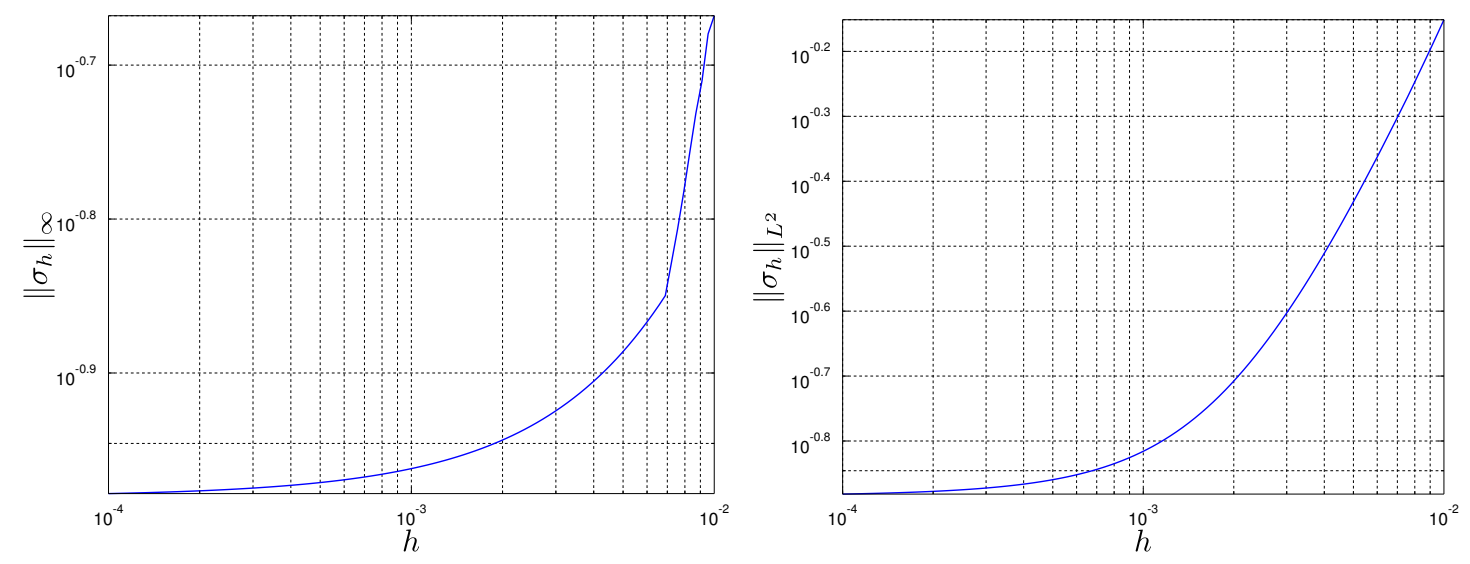

Figure 6: Evolution of the norm of the sliding variable $\sigma_{h}$ as a function of $h$ in a logarithmic scale $\left(\infty-\right.$ norm left, $L^{2}$-norm right).

\subsection{Control in the continuous/discrete setting}

In this case we consider again the two-link planar elbow manipulator with the same model and parameters as in the previous subsection. The main difference is that in this setting we take into account the continuous-time evolution of the plant given by (8) and the feedback loop is implemented using a stepwise controller obtained from the discretetime scheme (28)-(29). Notice that in this case a new disturbance appears due to the lack of knowledge about the exact discrete-time model of the nonlinear process. This last fact appears in contrast to the linear case, in which the user can obtain with the zero-order-hold discretization an exact discrete-time model. The performed simulations allow us to see how this new uncertainty is expressed in terms of chattering when the sampling time $h>0$ is big enough. In all the simulations of this subsection we maintain the same gains as before, except for $\gamma$ which is changed into $\gamma=0.5$. The signals obtained in simulation are shown in Figures 7-9 for several sampling times. Notice that in this setting the discrete-time sliding phase, $\hat{\sigma}_{k}=0$ for all $k \geq k^{*}$ for some $k^{*}<+\infty$, cannot be reached (even with precise knowledge of system parameters) because of the discretization error. This last fact induces an error in the set-valued input, which explains the appearance of chattering which was absent in the discrete/discrete setting. It is also worth to mention that this numerical chattering appears when $h=10^{-2}$ (Figure 7 ) and it vanishes for smaller sampling periods. The plots in Figure 6 depict how the norm of the sliding variable $\sigma_{h}$, associated to the continuous/discrete setting, evolves as a function of the sampling time $h>0$. In the simulations we consider two norms: the $\infty$-norm and the $L^{2}$-norm. From Figure 6 we can see that the order of convergence is not fixed and moreover, it tends to zero as $h$ decreases to zero. Finally, in order to have a frame of reference in the use of the implicit discretization, we present the case when the controller is discretized in an explicit way, i.e., (29) is replaced by,

$$
-u_{k} \in K_{\sigma} \sigma_{k}+\gamma \partial \Phi\left(\sigma_{k}\right) \text {. }
$$

Notice that in the explicit case there is no necessity of the scheme (31) since the variable $\sigma_{k}$ is assumed to be known at time $t_{k}$. The plots in Figures 10-11 show how the numerical chattering effect appears in the input and in the output of the closed-loop system with the sampling times $h=10^{-3}$ and $h=10^{-4}$ respectively, when the explicit method is used. On the contrary with the implicit controller chattering is almost suppressed for the same values of $h$, see Figures 8-9. Moreover, comparing the plots in Figure 8 against the plots in Figure 11, we see that the error in the sliding variable $\sigma_{h}$ and the chattering effect in both the input $\tau_{h}$ and the output $\sigma_{h}$, is much bigger with the explicit algorithm (75), 

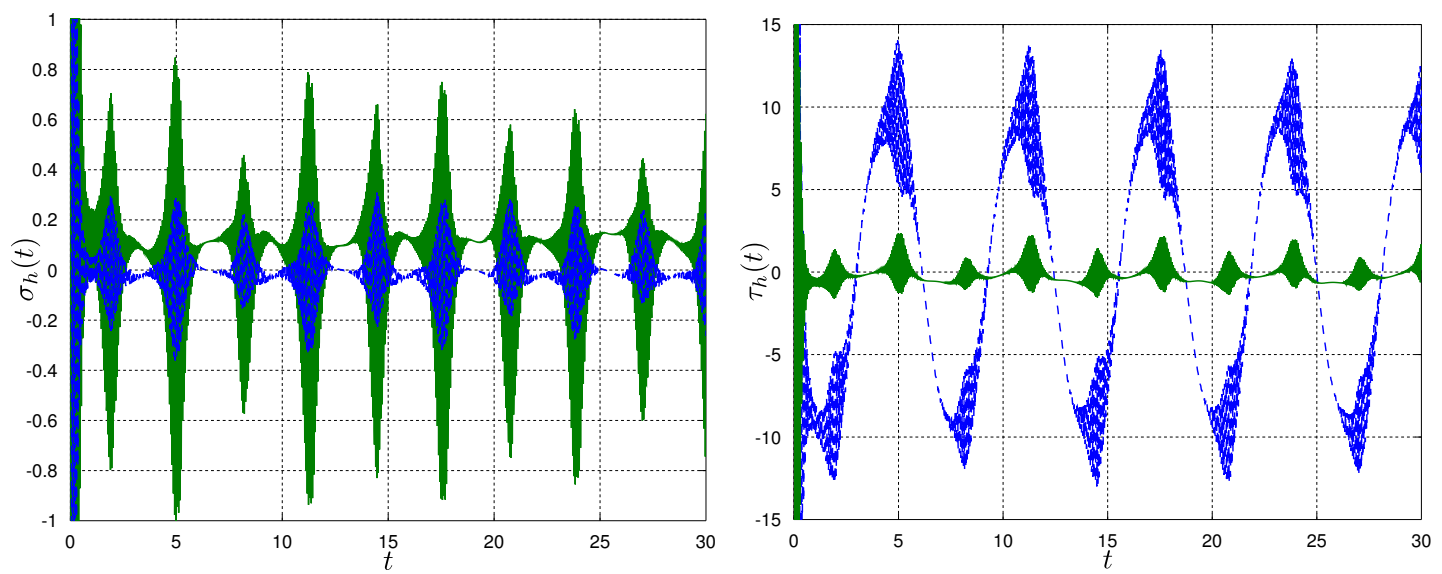

Figure 7: Evolution of the sliding variable $\sigma_{h}$ (left) and the control input $\tau_{h}$ (right) for the closed-loop system (8) (28) (29) with sampling time $h=10^{-2} s$.
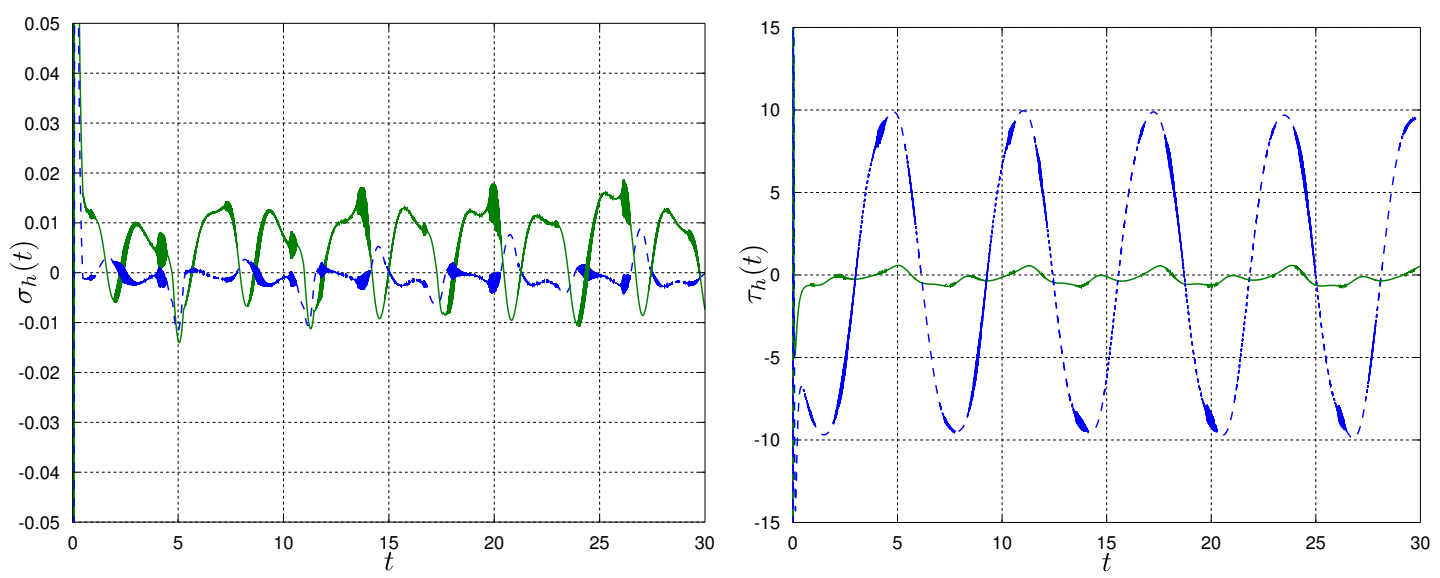

Figure 8: Evolution of the sliding variable $\sigma_{h}$ (left) and the control input $\tau_{h}$ (right) for the closed-loop system (8) (28) (29) with sampling time $h=10^{-3} s$.
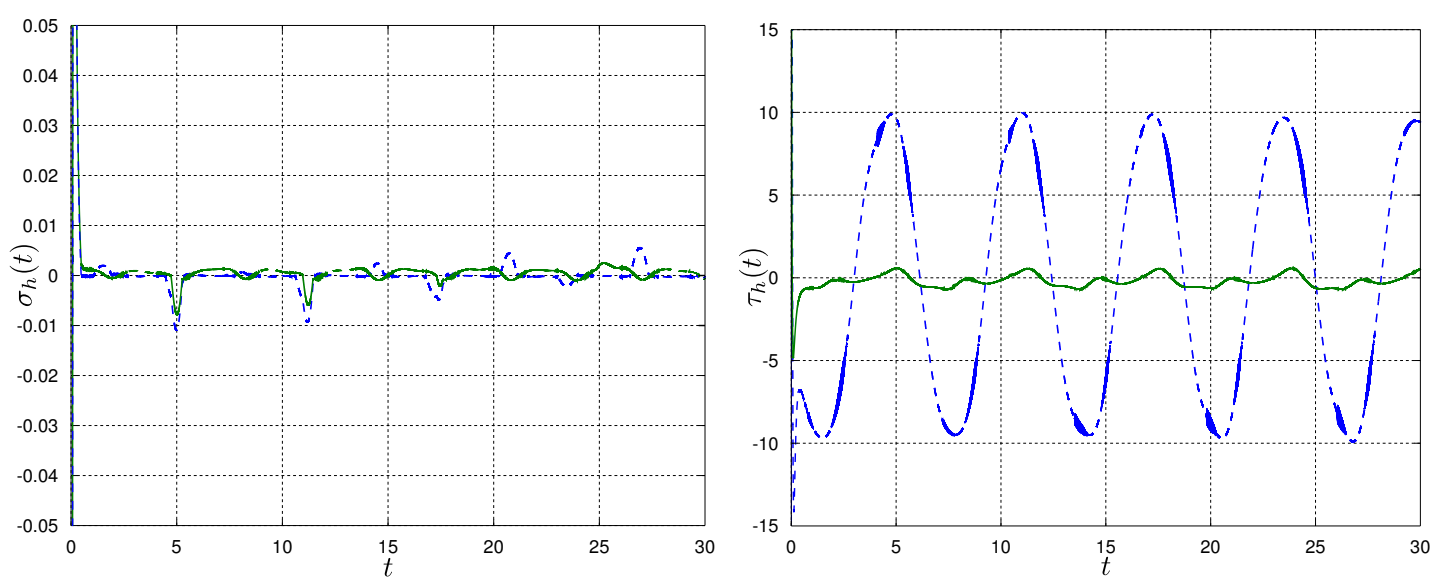

Figure 9: Evolution of the sliding variable $\sigma_{h}$ (left) and the control input $\tau_{h}$ (right) for the closed-loop system (8) (28) (29) with sampling time $h=10^{-4} s$. 

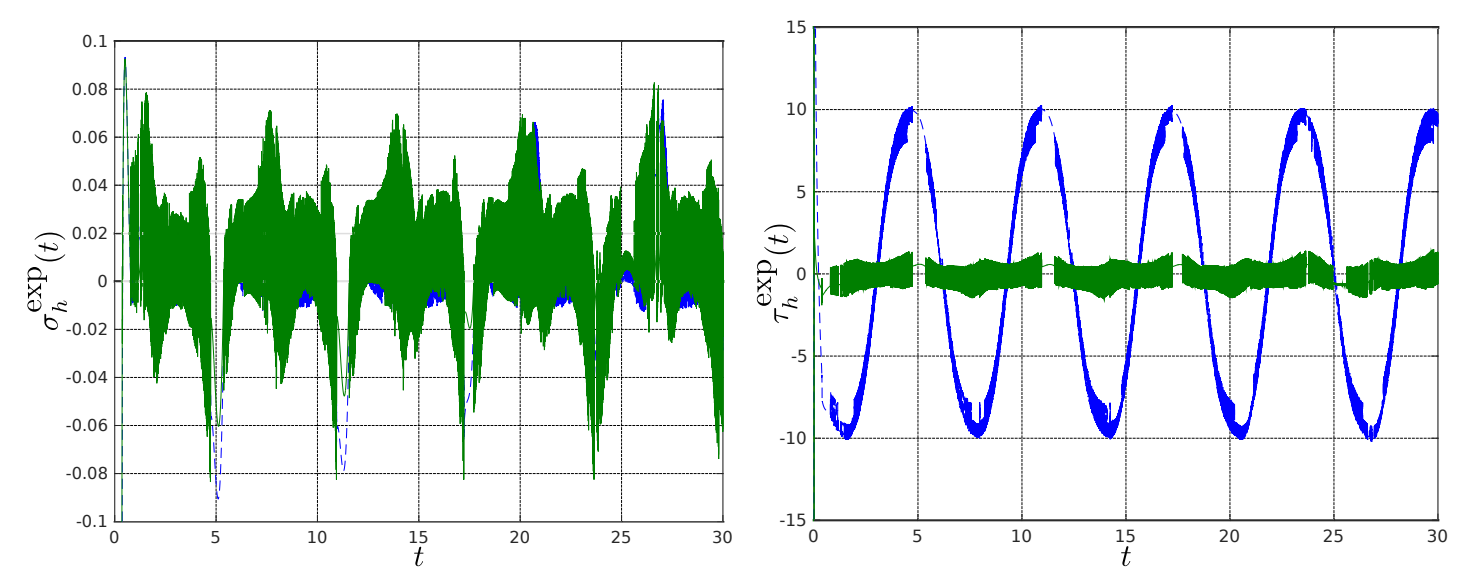

Figure 10: Evolution of sliding variable (left) and the control input(right) for the closedloop system (8) (28) (75) with sampling time $h=10^{-3} s$.
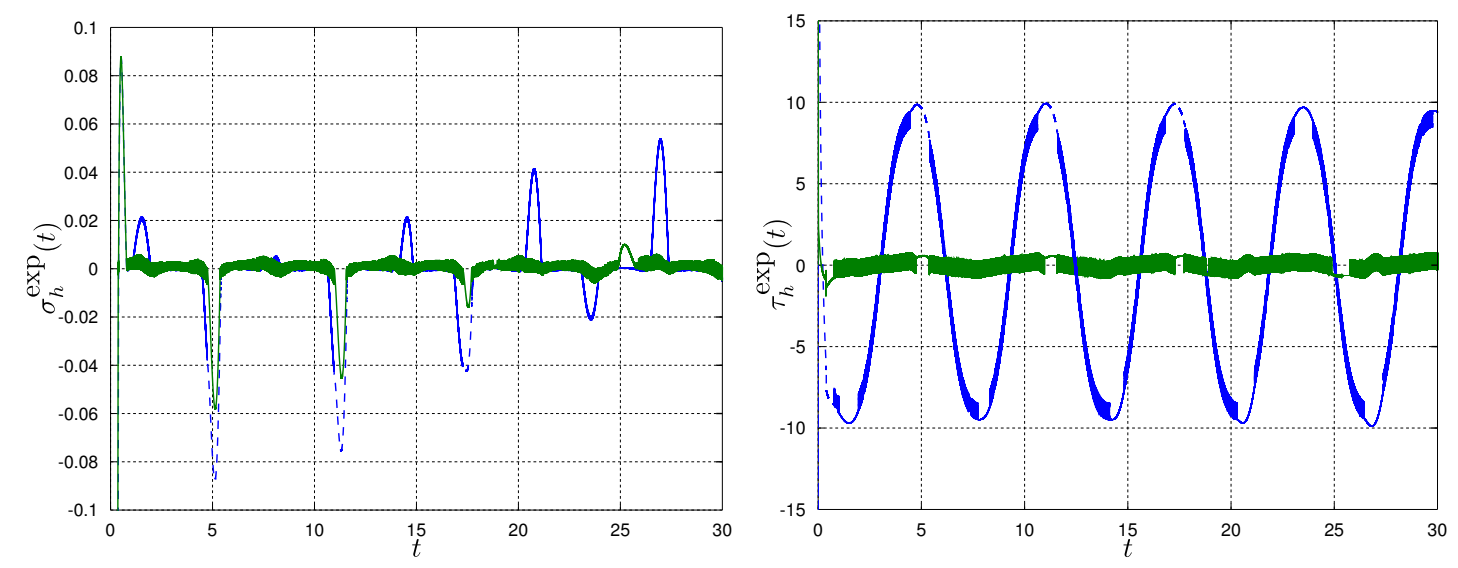

Figure 11: Evolution of the sliding variable (left) and the control input (right) for the closed-loop system (8) (28) (75) with sampling time $h=10^{-4} s$.

even when the sampling time for the implicit algorithm is ten times bigger. Finally it is worth to mention that in the case when $h=10^{-2}$ the resulting closed-loop system with the explicit controller (75) shows an unstable behavior (a conclusion already drawn for different plants and controllers in $[19,26]$ ), while the implicit algorithm keeps bounded input and output (see Figure 7). Therefore, the implicit (discrete-time) controller (29) shows a better performance when it is compared to its explicit counterpart (75), since the former allows much smaller sampling rates and exhibits a significantly better chattering alleviation, together with smaller error amplitudes. This shows that the conclusions drawn in $[17,18,19]$ from experimental data, extend to the nonlinear case analysed in this article, with parametric uncertainties.

\section{Conclusions and further research}

The main objective of this article is the analysis of a family of implicit discrete-time setvalued sliding mode controllers, for trajectory tracking in fully actuated Euler-Lagrange systems. First the continuous-time controllers are studied, with exogenous disturbances and parametric uncertainties. Well-posedness together with stability results are established. Subsequently the analysis of the implicit discrete-time scheme is done obtaining interesting features for the closed-loop discrete-time system: finite-time convergence for the nominal (unperturbed) sliding variable, robustness against external and para- 
metric uncertainties, convergence of solutions of the discrete-time system to solutions of the continuous-time closed-loop system, and suppression of chattering. Moreover, we show how the implicit discretization solves the problem related to the selection of a value from the set-valued controller resulting in an input that is not of the bang-bang type (i.e., no chattering in the input is presented), contrary to explicit discretizations. Some simulations validate the theoretical results, and also allow to better understand the limitations of the proposed scheme. Future research lines may consist in the study of better plant discretization in order to improve the behavior and provide an analysis of the continuous plant/discrete controller closed-loop system.

\section{References}

[1] V. Acary and B. Brogliato. Implicit Euler numerical scheme and chattering-free implementation of sliding mode systems. Systems \& Control Letters, 59(5):284-293, 2010.

[2] V. Acary, B. Brogliato, and Y. V. Orlov. Chattering-free digital sliding-mode control with state observer and disturbance rejection. Automatic Control, IEEE Transactions on, 57(5):1087-1101, 2012.

[3] K. Addi, B. Brogliato, and D. Goeleven. A qualitative mathematical analysis of a class of linear variational inequalities via semi-complementarity problems: applications in electronics. Mathematical Programming, 126(1):31-67, 2011.

[4] S. Adly, B. Brogliato, and B. K. Le. Well-posedness, robustness, and stability analysis of a set-valued controller for Lagrangian systems. SIAM Journal on Control and Optimization, 51(2):1592-1614, 2013.

[5] S. Adly, B. Brogliato, and B. K. Le. Implicit Euler time-discretization of a class of Lagrangian systems with set-valued robust controller. Journal of Convex Analysis, 23(1-2-3), 2016.

[6] J. Aubin and A. Cellina. Differential Inclusions: Set-Valued Maps and Viability Theory. Grundlehren der mathematischen Wissenschaften. Springer Berlin Heidelberg, 1984.

[7] J. Bastien and M. Schatzman. Numerical precision for differential inclusions with uniqueness. ESAIM: Mathematical Modelling and Numerical Analysis-Modélisation Mathématique et Analyse Numérique, 36(3):427-460, 2002.

[8] H. Bauschke and P. Combettes. Convex Analysis and Monotone Operator Theory in Hilbert Spaces. CMS Books in Mathematics. Springer New York, 2011.

[9] H. Brézis. Analyse Fonctionnelle: Théorie et Applications. Collection Mathématiques Appliquées pour la Maîtrise. Masson, Paris, 1993.

[10] B. Brogliato and D. Goeleven. Well-posedness, stability and invariance results for a class of multivalued Lur'e dynamical systems. Nonlinear Analysis: Theory, Methods and Applications, 74(1):195 - 212, 2011.

[11] B. Brogliato and A. Polyakov. Globally stable implicit Euler time-discretization of a nonlinear single-input sliding-mode control system. 54th IEEE Conference on Decision and Control, Osaka, Japan, December 2015. 
[12] B. Brogliato, R. Lozano, B. Maschke, and O. Egeland. Dissipative Systems Analysis and Control: Theory and Applications. Communications and Control Engineering. Springer Verlag London, 2nd edition, 2007.

[13] F. Facchinei and J. Pang. Finite-Dimensional Variational Inequalities and Complementarity Problems, volume II. Springer-Verlag, New York, 2003.

[14] Z. Galias and X. Yu. Euler's discretization of single input sliding-mode control systems. Automatic Control, IEEE Transactions on, 52(9):1726-1730, Sept 2007.

[15] D. Goeleven, D. Motreanu, Y. Dumont, and M. Rochdi. Variational and Hemivariational Inequalities: Theory, Methods and Applications, volume 1. Springer, 2003.

[16] J. B. Hiriart-Urruty and C. Lemaréchal. Convex Analysis and Minimization Algorithms I. Springer-Verlag, New York, 1993.

[17] O. Huber, V. Acary, B. Brogliato, and F. Plestan. Discrete-time twisting controller without numerical chattering: analysis and experimental results with an implicit method. Control Engineering Practice, 46(1): 129-141, January 2016.

[18] O. Huber, B. Brogliato, V. Acary, A. Boubakir, F. Plestan, and B. Wang. Experimental results on implicit and explicit time-discretization of equivalent-controlbased sliding mode control. In L. Fridman, J.-P. Barbot, and F. Plestan, editors, Recent Trends in Sliding Mode Control, The IET, ISBN: 978-1-78561-076-9, 2016.

[19] O. Huber, V. Acary, B. Brogliato. Lyapunov stability and performance analysis of the implicit discrete sliding mode control. Automatic Control, IEEE Transactions on, 2016, DoI: 10.1109/TAC.2015.2506991.

[20] R. Kelly, V. Santibáñez, and A. Loría. Control of Robot Manipulators in Joint Space. Springer-Verlag, 2005.

[21] H. Khalil. Nonlinear Systems. Prentice Hall, third edition, 2002.

[22] R. Kikuuwe, S. Yasukouchi, H. Fujimoto, M. Yamamoto. Proxy-based sliding mode control: A safer extension of PID position control. Robotics, IEEE Transactions on, 26(4): 670-683, 2010.

[23] A. N. Kolmogorov and S. V. Fomin. Elements of the Theory of Funtions and Functional Analysis, volume 1. Graylock Press, New York, 1957.

[24] A. J. Kurdila and M. Zabarankin. Convex Functional Analysis. Systems \& Control: Foundations \& Applications. Birkhäuser Verlag, Germany, 2005.

[25] P. Lancaster and M. Tismenetsky. The Theory of Matrices: With Applications. Academic Press, San Diego, CA, 1985.

[26] A. Levant. On fixed and finite time stability in sliding mode control. Proc. of 52th IEEE Conference on Decision and Control, 4260-4265, Firenze, Italy, December 2013.

[27] X. Li, N. Huang, and D. O'Regan. Differential mixed variational inequalities in finite dimensional spaces. Nonlinear Analysis: Theory, Methods 83 Applications, $72(9): 3875-3886,2010$. 
[28] F. A. Miranda-Villatoro and F. Castaños. Robust output regulation of strongly linear passive systems with multivalued maximally monotone controls. Submitted as Regular paper to Automatic Control, IEEE Transactions on, 2015.

[29] R. T. Rockafellar. Convex Analysis. Convex Analysis. Princeton University Press, 1970.

[30] R. T. Rockafellar and R. Wets. Variational Analysis. Die Grundlehren der mathematischen Wissenschaften in Einzeldarstellungen. Springer, 2009.

[31] W. Rugh. Linear System Theory. Prentice-Hall, second edition, 1996.

[32] J.-J. E. Slotine and W. Li. On the adaptive control of robot manipulators. The International Journal of Robotics Research, 6(3):49-59, 1987.

[33] J.-J. E. Slotine and W. Li. Adaptive manipulator control: A case study. Automatic Control, IEEE Transactions on, 33(11):995-1003, Nov 1988.

[34] J.-J. E. Slotine and S. S. Sastry. Tracking control of non-linear systems using sliding surfaces, with application to robot manipulators. International Journal of Control, 38(2):465-492, 1983.

[35] M. Spong, R. Ortega, and R. Kelly. Comments on "Adaptive manipulator control: a case study" by J. Slotine and W. Li. Automatic Control, IEEE Transactions on, 35(6):761-762, June 1990.

[36] A. Tanwani, B. Brogliato, and C. Prieur. On output regulation in state-constrained systems: An application to polyhedral case. In Proc. 19th IFAC World Congress, South Africa, pages 1513-1518, 2014.

[37] V. Utkin. Sliding Modes in Control and Optimization. Communications and Control Engineering. Springer-Verlag, 1992.

[38] V. Utkin, J. Guldner, and J. Shi. Sliding Mode Control in Electro-Mechanical Systems, volume 34. CRC press, 2009.

[39] A. J. van der Schaft. L2 - Gain and Passivity Techniques in Nonlinear Control. Communications and Control Engineering. Springer London, second edition, 2010.

[40] B. Wang, B. Brogliato, V. Acary, A. Boubakir, and F. Plestan. Experimental comparisons between implicit and explicit implementations of discrete-time sliding mode controllers: Toward input and output chattering suppression. Control Systems Technology, IEEE Transactions on, 23(5):2071-2075, Sept 2015.

[41] X. Yu, B. Wang, Z. Galias, G. Chen. Discretization effect on equivalent controlbased multi-input sliding-mode control systems. Automatic Control, IEEE Transactions on, 53(6): 1563-1569, July 2008. 